\title{
LIMIT THEOREMS FOR SOME SKEW PRODUCTS WITH MIXING BASE MAPS
}

\author{
YEOR HAFOUTA \\ INSTITUTE OF MATHEMATICS \\ HEBREW UNIVERSITY \\ JERUSALEM, ISRAEL
}

\begin{abstract}
We obtain central limit theorem, local limit theorems and renewal theorems for stationary processes generated by skew product maps $T(\omega, x)=\left(\theta \omega, T_{\omega} x\right)$ together with a $T$-invariant measure, whose base map $\theta$ satisfies certain topological and mixing conditions and the maps $T_{\omega}$ on the fibers are certain non-singular distance expanding maps. Our results hold true when $\theta$ is either a sufficiently fast mixing Markov shift with positive transition densities or a (non-uniform) Young tower with at least one periodic point and polynomial tails. The proofs are based on the random complex RuellePerron-Frobenius theorem from 13 applied with appropriate random transfer operators generated by $T_{\omega}$, together with certain regularity assumptions (as functions of $\omega$ ) of these operators. Limit theorems for deterministic processes whose distributions on the fibers are generated by Markov chains with transition operators satisfying a random version of the Doeblin condition will also be obtained. The main innovation in this paper is that the results hold true even though the spectral theory used in [1] does not seem to be applicable, and the dual of the Koopman operator of $T$ (with respect to the invariant measure) does not seem to have a spectral gap.
\end{abstract}

\section{INTRODUCTION}

Probabilistic limit theorems for deterministic dynamical systems and Markov chains is a well studied topic. Many results of this type are consequences of quasicompactness (i.e. spectral gap) of an appropriate Markov operator together with some perturbation theorem (see, for instance, [11, 15], 25] and [26]). The situation with limit theorems for random dynamical systems and Markov chains in random environment is more complicated, since, as opposed to the deterministic case, there is not only one underlying operator, but a family of random operators, so no spectral theory can be exploited. The central limit theorem (CLT) and large deviations theorem in this context can be obtained (see [22, 20, 21] and references therein) from the random real Ruelle-Perron-Frobenius (RPF) theorem (see, for instance, 21 and 23). Relying on this RPF theorem, limit theorems also follow from the spectral approach of [10. In [13] we proved a random complex RPF theorem and

Date: October 4, 2018.

2010 Mathematics Subject Classification. 60F05, 37H99, 60K37, 37D20, 37A25.

Key words and phrases. Markov chains; random environment; random dynamical systems; limit theorems; central limit theorem; local limit theorem; renewal theorem; 
used it to obtain (under certain conditions) a version of the Berry-Esseen theorem and the local central limit theorem for such processes in random environments.

Let $(\Omega, \mathcal{F}, P, \theta)$ be an ergodic invertible measure preserving system (MPS), $\mathcal{X}$ be a compact space and $T_{\omega}: \mathcal{X} \rightarrow \mathcal{X}$ be a random expanding non-singular transformation with respect to some probability measure $\mathbf{m}$. In this paper, under certain conditions, we will prove (annealed) limit theorems for stationary processes generated by the skew product map $T(\omega, x)=\left(\theta \omega, T_{\omega} x\right)$, together with a $T$-invariant measure $\boldsymbol{\mu}=\int \boldsymbol{\mu}_{\omega} d P(\omega)$ whose disintegrations $\boldsymbol{\mu}_{\omega}$ are certain random Gibbs measures. We will also obtain limit theorems for non-invertible MPS's in the case when $T$ preserves a product measure of the form $\boldsymbol{\mu}=P \times(\bar{h} \mathbf{m})$ for some continuous function $\bar{h}$. In [1] (see also references therein), the authors proved annealed limit theorems in the case when $T_{\omega}, T_{\theta \omega}, T_{\theta^{2} \omega}, \ldots$ are independent and the skew product map preserves a measure of the above product form. Our results hold true when the compositions $T_{\theta^{n-1} \omega} \circ T_{\theta^{n-2} \omega} \circ \cdots \circ T_{\omega}$ are taken along orbits $\left\{\theta^{k} \omega: k \geq 0\right\}$ of a map $\theta$ satisfying some mixing and topological conditions and having at least one periodic point, assuming that appropriate perturbations $\mathcal{L}_{i t}^{\omega}, t \in \mathbb{R}$ of the dual $\mathcal{L}_{0}^{\omega}$ (with respect to $\mathbf{m}$ ) of the Koopman operator $g \rightarrow g \circ T_{\omega}$ satisfy some regularity conditions (as functions of $\omega$ ) around one periodic orbit of $\theta$.

Quenched limit theorems, in our context, describe the asymptotic behaviour of the distribution the iterates $T_{\theta^{n-1} \omega} \circ T_{\theta^{n-2} \omega} \circ \cdots \circ T_{\omega} x, n \geq 1$, when $\omega$ is fixed (but rages over a set of full $P$-probability) and $x$ is distributed according to a random Gibbs measure. Often annealed limit theorems follow almost directly from the quenched ones just by integration over appropriate sets $\Gamma \subset \Omega$. We will use this approach (see Section 4) to prove an annealed CLT, but, for general $\theta$ 's, finer (annealed) limit theorems do not follow directly from the corresponding quenched limit theorems. For instance, in the local central limit theorem (LLT) the asymptotic behaviour as $n \rightarrow \infty$ of certain expectations multiplied by $\sqrt{n}$ is studied (see Theorem 2.7), which makes it impossible to choose appropriate sets $\Gamma$ which do not depend on $n$. The (annealed) renewal theorem also does not follow from a corresponding quenched limit theorem, since (see Theorem 2.8) it describes the asymptotic behaviour of certain series of expectations, and either way, to the best of my knowledge, no quenched renewal theorem has been proved in the setup of this paper.

In several circumstances annealed limit theorems follow from spectral theory of a single Markov operator together with some perturbation theorem. For instance, the results in [1] and [18 rely on the theory of quasi compact operators, where due to independence of the random maps the authors could exploit spectral properties of the averaged (Fourier) operators $\mathcal{A}_{i t}=\int \mathcal{A}_{i t}^{\omega} d P(\omega)$, obtained from integrating appropriate perturbations $\mathcal{A}_{i t}^{\omega}$ of the dual $\mathcal{A}_{0}^{\omega}$ of the Koopman operator $g \rightarrow g \circ T_{\omega}$ (with respect to an appropriate measure). In our situation the maps $T_{\omega}, T_{\theta \omega}, T_{\theta^{2} \omega}, \ldots$ are not independent, so there is no connection between the iterates of the averaged Fourier operators and the average $\int \mathcal{A}_{i t}^{\omega, n} d P(\omega)$ of the iterates $\mathcal{A}_{i t}^{\omega, n}=\mathcal{A}_{i t}^{\theta^{n-1} \omega_{\circ}} \cdots \circ \mathcal{A}_{i t}^{\theta \omega} \circ A_{i t}^{\omega}$ of the random Fourier operators. Another example is the case when the map $\theta$ is distance expanding. In this case the skew product map $T$ is also distance expanding (since $T_{\omega}$ 's are), and so limit theorems for stationary sums generated by $T$ follow from the spectral theory of the dual $\mathcal{L}$ of the Koopman operator $g \rightarrow g \circ T$. For instance, $\theta$ can be a topologically mixing subshift of finite type (see [3]) or a uniform Young tower (see 28, and [5]), but our results hold true 
for certain non-distance expanding maps such as (non-uniform) Young towers (see 29] and [5]) and uncountable Markov shifts.

In order to overcome the inapplicability of the spectral theory described in the previous paragraph, we will first apply the random complex RPF theorem from 13. After applying this RPF theorem, the main difficulty in proving the LLT and renewal theorem arises in obtaining appropriate (at least polynomial) decay of the integrals $\int\left\|\mathcal{A}_{i t}^{\omega, n}\right\| d P(\omega), t \neq 0$ as $n \rightarrow 0$. When $\omega$ is fixed, we obtained in [13] certain quenched LLT and Berry-Esseen theorem by showing that the operator norms $\left\|\mathcal{A}_{i t}^{\omega, n}\right\|, t \neq 0$ decay appropriately to 0 as $n \rightarrow \infty$. The problem in obtaining corresponding estimates of $\int\left\|\mathcal{A}_{i t}^{\omega, n}\right\| d P(\omega)$ by integration is that the rate of convergence of $\left\|\mathcal{A}_{i t}^{\omega, n}\right\|, t \neq 0$ to 0 , in general, is not uniform in $\omega$ as it depends, among other things, on certain almost sure limit theorems (e.g. on ergodic theorems). We will show that under appropriate mixing and topological conditions we can control, uniformly for $t$ 's belonging to compact sets $J$ not containing the origin, the order in $n$ of $\left\|\mathcal{A}_{i t}^{\omega, n}\right\|$ on sets $\Gamma_{n}=\Gamma_{n}(J)$, so that $1-P\left(\Gamma_{n}\right)$ decays polynomially fast to 0 . The arguments in the proof of these estimates can be viewed as averaged ("annealed") version of the periodic point approach from [13] (see Section 2.10 and Chapter 7 there).

Our results hold true when $\theta$ is the two sided Markov shift generated by a sufficiently fast mixing stationary Markov chain $\zeta_{n}, n \geq 0$ with positive transition densities and initial distribution assigning positive mass to open sets. In this case $\Omega=\mathcal{Y}^{\mathbb{Z}}$, where $\mathcal{Y}$ is the state space of the chain, and periodic points have the form $\bar{a}=(\ldots, a, a, a, \ldots), \bar{a}=\left(a_{i}\right)_{i=0}^{n_{0}-1} \in \mathcal{Y}^{n_{0}}$. In fact, our conditions will also be satisfied when the shift is generated by a stationary and sufficiently fast mixing process so that for some periodic $\bar{a}$ we have $P\left(\zeta_{i+(j-1) n_{0}} \in A_{j, i} ; 0 \leq i<n_{0}, 1 \leq j \leq s\right)>0$ for any open sets $A_{i, j}$ so that $a_{i} \in A_{i, j}$ for all $i$ and $j$. When (almost) all the $T_{\omega}$ 's preserve the same absolutely continuous (with respect to $\mathbf{m}$ ) measure $\kappa$, then our results also hold true for non-invertible $\theta$ 's such as (non-uniform) Young towers (see [29] and [5]) with at least one periodic point and exponential tails. In fact, we obtain also results for compositions of random maps having the form $T_{\omega}(x)=\boldsymbol{T}_{\mathcal{O}(\omega)}(x)$, where $\boldsymbol{T}_{q}(x)$ is a Hölder continuous function of the variable $q \in \Omega^{\mathbb{N}}, \theta$ is the shift map and $\mathcal{O}(\omega)=\left\{\theta^{n} \omega: n \geq 0\right\}$ is the orbit of a topologically mixing subshift of finite type or a Young tower with the properties described above.

\section{Preliminaries and main Results}

Our setup consists of a complete probability space $(\Omega, \mathcal{F}, P)$ together with a $P$ preserving ergodic transformation $\theta: \Omega \rightarrow \Omega$ and of a compact metric space $(\mathcal{X}, \rho)$ together with the Borel $\sigma$-algebra $\mathcal{B}$. Set $\mathcal{E}=\Omega \times \mathcal{X}$ and let

$$
\left\{T_{\omega}: \mathcal{X} \rightarrow \mathcal{X}, \omega \in \Omega\right\}
$$

be a collection of continuous bijective maps between $\mathcal{X}$ to itself so that the map $(\omega, x) \rightarrow T_{\omega} x$ is measurable with respect to $\mathcal{F} \times \mathcal{B}$. Consider the skew product transformation $T: \mathcal{E} \rightarrow \mathcal{E}$ given by

$$
T(\omega, x)=\left(\theta \omega, T_{\omega} x\right) .
$$

For any $\omega \in \Omega$ and $n \in \mathbb{N}$ consider the $n$-th step iterates $T_{\omega}^{n}$ given by

$$
T_{\omega}^{n}=T_{\theta^{n-1} \omega} \circ \cdots \circ T_{\theta \omega} \circ T_{\omega}: \mathcal{X} \rightarrow \mathcal{X} .
$$


Then $T^{n}(\omega, x)=\left(\theta^{n} \omega, T_{\omega}^{n} x\right)$. The main results of this paper are probabilistic limit theorems for random variables of the form $S_{n} u(\omega, x)$, where $S_{n}=\sum_{j=0}^{n-1} u$ 。 $T^{n}, u=u(\omega, x)$ is a function satisfying certain regularity conditions and $(\omega, x)$ is distributed according to some special $T$-invariant probability measure $\boldsymbol{\mu}$. Our additional requirements concerning the family of maps $\left\{T_{\omega}: \omega \in \Omega\right\}$ are collected in the following assumptions which are similar to 24].

2.1. Assumption (Topological exactness). There exist a constant $\xi>0$ and a random variable $n_{\omega} \in \mathbb{N}$ such that $P$-a.s.,

$$
T_{\omega}^{n_{\omega}}(B(x, \xi))=\mathcal{X} \text { for any } x \in \mathcal{X}
$$

where for any $x \in \mathcal{X}$ and $r>0, B(x, r)$ denotes a ball in $\mathcal{X}$ around $x$ with radius $r$.

2.2. Assumption (The pairing property). There exist random variables $\gamma_{\omega}>1$ and $D_{\omega}$ such that $P$-a.s. for any $x, x^{\prime} \in \mathcal{X}$ with $\rho\left(x, x^{\prime}\right)<\xi$ we can write

$$
T_{\omega}^{-1}\{x\}=\left\{y_{1}, \ldots, y_{k}\right\} \text { and } T_{\omega}^{-1}\left\{x^{\prime}\right\}=\left\{y_{1}^{\prime}, \ldots, y_{k}^{\prime}\right\}
$$

where $\xi$ is specified in Assumption 2.1.

$$
k=k_{\omega, x}=\left|T_{\omega}^{-1}\{x\}\right| \leq D_{\omega},
$$

$|\Gamma|$ is the cardinality of a finite set $\Gamma$ and

$$
\rho\left(y_{i}, y_{i}^{\prime}\right) \leq\left(\gamma_{\omega}\right)^{-1} \rho\left(x, x^{\prime}\right)
$$

for any $1 \leq i \leq k$.

Next, let $\alpha \in(0,1]$. For any measurable function $g: \mathcal{E} \rightarrow \mathbb{C}$ and $\omega \in \Omega$ consider the function $g_{\omega}: \mathcal{X} \rightarrow \mathbb{C}$ given by $g_{\omega}(x)=g(\omega, x)$. Set

$$
\begin{gathered}
v_{\alpha, \xi}\left(g_{\omega}\right)=\inf \left\{R:\left|g_{\omega}(x)-g_{\omega}\left(x^{\prime}\right)\right| \leq R \rho^{\alpha}\left(x, x^{\prime}\right) \text { if } \rho\left(x, x^{\prime}\right)<\xi\right\} \\
\text { and }\left\|g_{\omega}\right\|_{\alpha, \xi}=\left\|g_{\omega}\right\|_{\infty}+v_{\alpha, \xi}\left(g_{\omega}\right)
\end{gathered}
$$

where $\|\cdot\|_{\infty}$ is the supremum norm and $\rho^{\alpha}\left(x, x^{\prime}\right)=\left(\rho\left(x, x^{\prime}\right)\right)^{\alpha}$. The norms $\left\|g_{\omega}\right\|_{\alpha, \xi}$ are $\mathcal{F}$-measurable as a consequence of Lemma 5.1.3 in [13. We denote by $\mathcal{H}^{\alpha, \xi}$ the space of all functions $f: \mathcal{X} \rightarrow \mathbb{C}$ so that $\|f\|_{\alpha, \xi}<\infty$.

Let $\phi, u: \mathcal{E} \rightarrow \mathbb{R}$ be two measurable functions so that $P$-a.s. we have $\phi_{\omega}, u_{\omega} \in$ $\mathcal{H}^{\alpha, \xi}$. Let $z \in \mathbb{C}$ and consider the transfer operators $\mathcal{L}_{z}^{\omega}, \omega \in \Omega$ which map functions on $\mathcal{X}$ to functions on $\mathcal{X}$ by the formula

$$
\mathcal{L}_{z}^{\omega} g(x)=\sum_{y \in T_{\omega}^{-1}\{x\}} e^{\phi_{\omega}(y)+z u_{\omega}(y)} g(y) .
$$

For any $n \geq 1$ set

$$
\mathcal{L}_{z}^{\omega, n}=\mathcal{L}_{z}^{\theta^{n-1} \omega} \circ \cdots \circ \mathcal{L}_{z}^{\theta \omega} \circ \mathcal{L}_{z}^{\omega} .
$$

Then $\mathcal{L}_{z}^{\omega, n} g(x)=\sum_{y \in\left(T_{\omega}^{n}\right)^{-1}\{x\}} e^{S_{n}^{\omega} \phi(y)+z S_{n}^{\omega} u(y)} g(y)$, where $S_{n}^{\omega} \psi=\sum_{j=0}^{n-1} \psi_{\theta^{j} \omega} \circ T_{\omega}^{j}$ for any $\psi: \mathcal{E} \rightarrow \mathbb{C}$. Henceforth we will rely on

2.3. Assumption. (i) The random variables $n_{\omega}, D_{\omega},\left\|\phi_{\omega}\right\|_{\alpha, \xi}$ and $\left\|u_{\omega}\right\|_{\alpha, \xi}$ are bounded and $\gamma_{\omega}-1$ is bounded from below by some positive constant.

(ii) The transfer operators $\mathcal{L}_{z}^{\omega}$ are measurable, namely the map $(\omega, x) \rightarrow \mathcal{L}_{z}^{\omega} g_{\omega}(x)$ is measurable, for any measurable function $g: \mathcal{E} \rightarrow \mathbb{C}$. 
Since $\phi$ and $u$ are measurable, $\mathcal{L}_{z}, z \in \mathbb{C}$ are measurable when for each $y \in \mathcal{X}$, the map $(\omega, x) \rightarrow \mathbb{I}_{T_{\omega}^{-1}\{x\}}(y)$ is measurable with respect to $\mathcal{F} \times \mathcal{B}$, where $\mathbb{I}_{C}$ is the indicator function of a set $C$. Under Assumption 2.3 (i) we have $\mathcal{L}_{z}^{\omega, n}\left(\mathcal{H}_{\omega}^{\alpha, \xi}\right) \subset \mathcal{H}_{\theta^{n} \omega}^{\alpha, \xi}$ and the corresponding operator norm satisfies $\left\|\mathcal{L}_{i t}^{\omega, n}\right\|_{\alpha, \xi} \leq B(1+|t|)$ where $B$ is some constant (see Lemma 5.6.1 in [13] and Section 3.2).

2.1. Results for invertible base maps. We assume here that the measure preserving system $(\Omega, \mathcal{F}, P, \theta)$ is invertible. Let $\boldsymbol{\mu}=\int \boldsymbol{\mu}_{\omega} d P(\omega)$ be the $T$ invariant Gibbs measure associated with $T_{\omega}$ and $\phi_{\omega}$. Namely, $\boldsymbol{\mu}_{\omega}$ has the form $\boldsymbol{\mu}_{\omega}=\mathbf{h}_{\omega}(0) \boldsymbol{\nu}_{\omega}(0)$ where $\mathbf{h}_{\omega}(0)$ and $\boldsymbol{\nu}_{\omega}(0)$ are members of the (random) RPF triplets of the family $\mathcal{L}_{0}^{\omega}, \omega \in \Omega$, as described in Section 3. Note that the measure $\boldsymbol{\mu}$ coincides with the Gibbs measures in the setup of either 24] or 23. Recall that under our conditions (see [22]), the limit $\sigma^{2}=\lim _{n \rightarrow \infty} \frac{1}{n} \operatorname{var}_{\mu_{\omega}(0)} S_{n}^{\omega} u$ exists $P$ a.s. and it does not depend on $\omega$. Moreover, $\sigma^{2}>0$ if and only if $\bar{u}_{\omega}=u_{\omega}-\boldsymbol{\mu}_{\omega}\left(u_{\omega}\right)$ does not admit a coboundary representation of the form

$$
\bar{u}_{\omega}=q_{\omega}-q_{\theta \omega} \circ T_{\omega}
$$

for some function $q=q(\omega, x) \in L^{2}(\mathcal{E}, \mathcal{F} \times \mathcal{B}, \boldsymbol{\mu})$. Consider the functions $S_{n} u: \mathcal{E} \rightarrow$ $\mathbb{R}$ given by $S_{n} u=\sum_{j=0}^{n-1} u \circ T^{n}$. We first state

2.4. Theorem. Suppose that assumption 2.3 is satisfied and that $\boldsymbol{\mu}_{\omega}\left(u_{\omega}\right)=$ $\int u_{\omega} d \boldsymbol{\mu}_{\omega}=0, P$-a.s. Then the CLT holds true, namely for any $r \in \mathbb{R}$,

$$
\lim _{n \rightarrow \infty} \boldsymbol{\mu}\left\{(\omega, x): n^{-\frac{1}{2}} S_{n} u(\omega, x) \leq r\right\}=\frac{1}{\sqrt{2 \pi} \sigma} \int_{-\infty}^{r} e^{-\frac{t^{2}}{2 \sigma^{2}}} d t
$$

where when $\sigma=0$ the above right hand side is defined to be 1 if $r \geq 0$ and 0 if $r<0$.

Note that Theorem 2.4 follows from the quenched CLT in 22] (via integration), but for readers' convenience we will prove it using the random complex RPF theorem. The proof will be very short and rely on the arguments in the proof of the quenched Berry-Esseen theorem in [13. Our main interest in this paper is in finer limit theorems such the LLT and renewal theorem, which, in general, do not follow from the quenched ones (when exist), but the above CLT is needed in Theorem 2.7 below.

In order to obtain LLT's and renewal theorems we will need the following

2.5. Assumption. The space $\Omega$ is a topological space, $\mathcal{F}$ contains in the corresponding Borel $\sigma$-algebra and $\theta$ has a periodic point, namely there exist $\omega_{0} \in \Omega$ and $n_{0} \in \mathbb{N}$ so that $\theta^{n_{0}} \omega_{0}=\omega_{0}$. Moreover, for any compact interval $J$ the maps $\omega \rightarrow \mathcal{L}_{i t}^{\omega}$ are uniformly continuous (with respect to the operator norm $\|\cdot\|_{\alpha, \xi}$ ) at the points $\theta^{j} \omega_{0}, 0 \leq j<n_{0}$ when $t$ ranges over $J$.

The condition about continuity of $\omega \rightarrow \mathcal{L}_{i t}^{\omega}$ holds true, for instance, when the maps $\omega \rightarrow \phi_{\omega}, u_{\omega} \in \mathcal{H}^{\alpha, \xi}$ are continuous at the points $\theta^{j} \omega_{0}, 0 \leq j<n_{0}$ and $\omega \rightarrow T_{\omega}$ is either locally constant there or is continuous at these points with respect to an appropriate topology.

Next, for any $n \geq 1$ consider the function $V_{n}: \Omega \rightarrow \mathbb{R}$ given by $V_{n}(\omega)=$ $\operatorname{var}_{\boldsymbol{\mu}_{\omega}} S_{n}^{\omega} u=\boldsymbol{\mu}_{\omega}\left(S_{n}^{\omega} \bar{u}\right)^{2}$. We will also use the following probabilistic growth type conditions. 
2.6. Assumption. The asymptotic variance $\sigma^{2}$ is positive. Moreover, there exist $\beta>0$ and $c_{1}, c_{2}>0$ so that for any $n \geq 1$,

$$
P\left\{\omega: V_{n}(\omega) \leq c_{1} n\right\} \leq \frac{c_{2}}{n^{\beta}} .
$$

Furthermore, for any $s>0$ and neighborhoods $B_{\theta^{j} \omega_{0}}$ of $\theta^{j} \omega_{0}, j=0,1, \ldots, n_{0}-1$ the set $B_{\omega_{0}, n_{0}, s}=\bigcap_{i=0}^{s n_{0}-1} \theta^{-i} B_{\theta^{i} \omega_{0}}$ satisfies that for any $n \geq 1$,

$$
P\left\{\omega: \sum_{j=0}^{n-1} \mathbb{I}_{B_{\omega_{0}, n_{0}, s}}\left(\theta^{j} \omega\right) \leq c n\right\} \leq \frac{d}{n^{\beta}}
$$

where $c$ and $d$ are positive constants which depend only on $s, \omega_{0}$ and $n_{0}$ and $\mathbb{I}_{B}$ is the indicator function of a set $B$.

When $\mathcal{X}$ is a $C^{2}$-compact Riemanian manifold, $T_{\omega}$ are certain $C^{2}$ random endomorphisms and $e^{-\phi_{\omega}}$ is the corresponding Jacobian then $\nu_{\omega}(0)=\mathbf{m}$ (see Theorem 2.2 in [23]), where $\mathbf{m}$ is the (normalized) volume measure. In these circumstances, in Proposition 5.1, we will show that condition (2.7) is satisfied when polynomial concentration inequalities of the form (5.2) hold true. In fact, the above also holds true when all $T_{\omega}$ 's are nonsingualr with respect to some measure $\mathbf{m}$ (see Proposition 3.2) and $\phi_{\omega}=-\ln \left(\frac{d\left(T_{\omega}\right)_{*} \mathbf{m}}{d \mathbf{m}}\right)$. When $T_{\omega}$ satisfy certain regularity conditions as function of $\omega$, in Propositions 5.3 we show that conditions (5.2) and (2.8) are satisfied when $\theta$ is a (non-uniform) Young tower (see [29] and [5]) with one at least one periodic point and polynomial tails. Of course, Young towers are not invertible, but in Section 2.2 we explain how to obtain results under Assumption 2.6 for non-invertible maps. In Propositions 5.4 and 5.5 we show that the latter conditions also hold true when $\theta$ is a Markov shift generated by a Markov chain with positive transition densities satisfying the Doeblin condition. We refer the readers' to Section 5 for precise statements and full details.

As usual (see, for instance, [1] and [15]), in order to present the local limit theorem and the renewal theorem we will distinguish between two cases. Under Assumption 2.5. we call the case a non-lattice one if the function $S_{n_{0}}^{\omega_{0}} u=\sum_{j=0}^{n-1} u_{\theta^{j} \omega_{0}} \circ T_{\omega_{0}}^{j}$ is non-arithmetic (aperiodic) with respect to the map $\tau=T_{\omega_{0}}^{n_{0}}$ in the classical sense of [15, namely the spectral radius (with respect to the norm $\|\cdot\|_{\alpha, \xi}$ ) of the operators $R_{i t}=\mathcal{L}_{i t}^{\omega_{0}, n_{0}}, t \neq 0$ are strictly less than 1 . We call the case a lattice one if there exists $h>0$ such that $P$-a.s. the function $u_{\omega}$ takes values on the lattice $h \mathbb{Z}=\{h k: k \in \mathbb{Z}\}$ and the spectral radius of the operators $R_{i t}, t \in\left(-\frac{2 \pi}{h}, \frac{2 \pi}{h}\right) \backslash\{0\}$ are strictly less than 1 . We refer the readers to [15] for conditions equivalent to the above lattice and non-lattice conditions.

Next, for any $r>0$ let $C_{r \downarrow}(\mathbb{R})$ be the space of all continuous functions $g: \mathbb{R} \rightarrow \mathbb{C}$ so that $\lim _{x \rightarrow \infty} x^{r} g(x)=0$.

2.7. Theorem (Local limit theorem). Suppose that Assumptions [2.3, 2.5 and 2.6 hold true, where in the last assumption we require that $\beta>\frac{1}{2}$. Moreover, assume that $\boldsymbol{\mu}_{\omega}(0)\left(u_{\omega}\right)=\int u_{\omega} d \boldsymbol{\mu}_{\omega}(0)=0, P$-a.s. Then for any $g \in C_{2 \downarrow}(\mathbb{R})$,

$$
\lim _{n \rightarrow \infty} \sup _{a \in \mathcal{T}_{h}}\left|\sigma \sqrt{2 \pi n} \boldsymbol{\mu}\left(g\left(S_{n} u-a\right)\right)-\kappa_{h}(g) e^{-\frac{a^{2}}{2 n \sigma^{2}}}\right|=0
$$

where in the lattice case $\kappa_{h}$ is the measure assigning unit mass to each member of the lattice $\mathcal{T}_{h}=h \mathbb{Z}$, while in the non-lattice case we set $h=0$ and take $\kappa_{0}$ and $\mathcal{T}_{0}$ to be the Lebesgue measure and the real line, respectively. 
2.8. Theorem (Renewal theorem). Suppose that Assumptions 2.3. 2.5 and 2.6 hold true, where in the last assumption we require that $\beta>1$. Moreover, assume that $\boldsymbol{\mu}_{\omega}(0)\left(u_{\omega}\right)=\gamma>0$ does not depend on $\omega$. Let $f: \mathcal{E} \rightarrow \mathbb{R}$ be a positive function so that $f_{\omega} \in \mathcal{H}_{\alpha, \xi}^{\omega}, \boldsymbol{\mu}_{\omega}(0)\left(f_{\omega}\right)=\boldsymbol{\mu}(f)$ does not depend on $\omega$ and that $\left\|f_{\omega}\right\|_{\alpha, \xi} \in L^{p}(\Omega, \mathcal{F}, P)$ for some $1<p \leq \infty$ so that $\beta\left(1-\frac{1}{p}\right)>1$. For any Borel measurable set $B \subset \mathbb{R}$ set

$$
U(B)=U_{\mu, f}(B)=\sum_{n \geq 1} \boldsymbol{\mu}\left(f \mathbb{I}_{B}\left(S_{n} u\right)\right)
$$

where $\mathbb{I}_{B}$ is the indicator function of the set $B$. Then in both lattice and non-lattice cases $U$ is a Radon measure on $\mathbb{R}$ so that $\int|g| d U<\infty$ for any $C_{4 \downarrow}(\mathbb{R})$. Moreover, for any function $g \in C_{4 \downarrow}(\mathbb{R})$,

$$
\lim _{a \rightarrow-\infty} U\left(g_{a}\right)=0 \text { and } \lim _{a \rightarrow \infty} U\left(g_{a}\right)=\frac{\boldsymbol{\mu}(f)}{\gamma} \kappa_{h}(g)
$$

where $g_{a}(x)=g(x-a)$, and in the lattice case $\kappa_{h}$ is the measure assigning unit mass to each member of the lattice $h \mathbb{Z}$, while in the non-lattice case we set $h=0$ and take $\kappa_{0}$ to be the Lebesgue measure.

2.2. The non-invertible case. Suppose that $(\Omega, \mathcal{F}, P, \theta)$ is ergodic and not necessarily invertible. Henceforth, we will assume that all the maps $T_{\omega}$ are non-singular with respect to some probability measure $\mathbf{m}$ on $\mathcal{X}$ so that $\operatorname{supp} \mathbf{m}=\mathcal{X}$, that $\phi_{\omega}=-\ln \left(\frac{d\left(T_{\omega}\right)_{*} \mathbf{m}}{d \mathbf{m}}\right)$ and that $P$-a.s. the map $T_{\omega}$ preserves a measure $\kappa$ of the form $\kappa=\bar{h} \mathbf{m}$, where $\bar{h}$ is some continuous nonnegative function on $\mathcal{X}$ which does not depend on $\omega$. The latter condition means that the skew product map $T(\omega, x)=\left(\theta \omega, T_{\omega} x\right)$ preserves the product measure $\boldsymbol{\mu}=P \times \kappa$, whose disintegrations $\boldsymbol{\mu}_{\omega}$ equal $\kappa$. Existence of such product measures was studied in [1] (see also references therein). Note that in [1] the authors considered independent maps $T_{\omega}, T_{\theta \omega}, T_{\theta^{2} \omega}, \ldots$, but the $T$-invariance of $\boldsymbol{\mu}$ defined above depends only on the distribution of $T_{\omega}$, and not on the dependencies between $T_{\theta^{j}}$ 's.

2.9. Theorem. The limit $\sigma^{2}=\lim _{n \rightarrow \infty} \frac{1}{n} \operatorname{var}_{\kappa} S_{n}^{\omega} u$ exists $P$-a.s. and it does not depend on $\omega$. Moreover, $\sigma^{2}>0$ if and only if $\bar{u}_{\omega}=u_{\omega}-\kappa\left(u_{\omega}\right)$ does not admit a coboundary representation of the form

$$
\bar{u}_{\omega}=q_{\omega}-q_{\theta \omega} \circ T_{\omega}
$$

for some function $q=q(\omega, x) \in L^{2}(\mathcal{E}, \mathcal{F} \times \mathcal{B}, \boldsymbol{\mu})$. Moreover, the $C L T$, the local limit theorem and the renewal theorem stated in Theorems 2.4, 2.7 and 2.8 hold true.

We remark that the condition that $\boldsymbol{\mu}_{\omega}\left(u_{\omega}\right)=\kappa\left(u_{\omega}\right)$ does not depend on $\omega$ is satisfied when $u_{\omega}$ is replaced with $\frac{u_{\omega}}{\kappa\left(u_{\omega}\right)}$. Since $\kappa\left(u_{\omega}\right)$ is Hölder continuous in $\omega$ when $u_{\omega}$ is, all the continuity Assumptions from 2.5 still hold true after this replacement. Theorem 2.9 is proved by applying Theorems $2.4,2.7$ and 2.8 with the natural invertible extension of $(\Omega, \mathcal{F}, P, \theta)$, see Section 4.1. Applying these theorems yields results even when $T_{\omega}$ 's do not preserve the same absolutely continuous measure $\kappa$, but then the conditions for the limit theorems to hold true (derived from the results in the extension) are not natural (see Remark 4.1). We also note that the situation described in Section 6.1 makes it possible to consider the case when $\phi_{\omega}=-\ln \left(\frac{d\left(T_{\omega}\right)_{*} \mathbf{m}}{d \mathbf{m}}\right)$ is only Hölder continuous on some pieces of $\mathcal{X}$ (e.g. when $\mathcal{X}$ is the unit circle and $T_{\omega}(x)=x^{m_{\omega}}$ for some $\left.m_{\omega} \in \mathbb{N}\right)$. 


\section{RANDOM COMPLEX RPF THEOREM AND THERMODYNAMIC FORMALISM CONSTRUCTIONS}

Suppose that $(\Omega, \mathcal{F}, P, \theta)$ is invertible. In this section we mainly collect several results from 13 which will be used in the proofs of the results stated in Section 2. Let $\left(\boldsymbol{\lambda}_{\omega}(z), \boldsymbol{h}_{\omega}(z), \boldsymbol{\nu}_{\omega}(z)\right)$ be the (random) RPF triplet of the family $\mathcal{L}_{z}^{\omega}, \omega \in \Omega$, obtained in Corollary 5.4.2 in [13]. Let the probability measure $\boldsymbol{\mu}_{\omega}=\boldsymbol{\mu}_{\omega}(0)$ be given by $\boldsymbol{\mu}_{\omega}=\boldsymbol{h}_{\omega}(0) d \boldsymbol{\nu}_{\omega}(0)$. Then the measure $\boldsymbol{\mu}=\boldsymbol{\mu}(0)=\int \boldsymbol{\mu}_{\omega} d P(\omega)$ is $T$ invariant. We refer the readers' to [24] and 23] for important properties of these measures (in the setups considered there). Consider the (transfer) operator $\mathcal{A}_{z}^{\omega}$ given by $\mathcal{A}_{z}^{\omega} g=\frac{\mathcal{L}_{z}^{\omega}\left(g \boldsymbol{h}_{\omega}(0)\right)}{\boldsymbol{\lambda}_{\omega}(0) \boldsymbol{h}_{\theta \omega}(0)}$, namely the transfer operator generated by $T_{\omega}$ and the function $\phi_{\omega}+\ln \mathbf{h}_{\omega}(0)-\ln \mathbf{h}_{\theta \omega} \circ T_{\omega}-\ln \boldsymbol{\lambda}_{\omega}(0)+z u_{\omega}$. Then the RPF theorem stated as Corollary 5.4.2 in [13] holds true also for $\mathcal{A}_{z}^{\omega}$, as stated in the following

3.1. Theorem. There is a (bounded) neighborhood $U \subset \mathbb{C}$ of 0 so that P-a.s. for any $z \in U$ there exists a triplet $\lambda_{\omega}(z), h_{\omega}(z)$ and $\nu_{\omega}(z)$ consisting of a nonzero complex number $\lambda_{\omega}(z)$, a complex function $h_{\omega}(z) \in \mathcal{H}_{\omega}^{\alpha, \xi}$ and a complex continuous linear functional $\nu_{\omega}(z)$ on $\mathcal{H}_{\omega}^{\alpha, \xi}$ such that

$$
\begin{array}{r}
\mathcal{A}_{z}^{\omega} h_{\omega}(z)=\lambda_{\omega}(z) h_{\theta \omega}(z),\left(\mathcal{A}_{z}^{\omega}\right)^{*} \nu_{\theta \omega}(z)=\lambda_{\omega}(z) \nu_{\omega}(z) \text { and } \\
\nu_{\omega}(z) h_{\omega}(z)=\nu_{\omega}(z) \mathbf{1}=1 .
\end{array}
$$

For any $z \in U$ the maps $\omega \rightarrow \lambda_{\omega}(z)$ and $(\omega, x) \rightarrow h_{\omega}(z)(x),(\omega, x) \in \mathcal{E}$ are measurable and the family $\nu_{\omega}(z)$ is measurable in $\omega$. When $z=t \in \mathbb{R}$ then $\lambda_{\omega}(t)>0$, the function $h_{\omega}(t)$ is strictly positive, $\nu_{\omega}(t)$ is a probability measure and the equality $\nu_{\theta \omega}(t)\left(\mathcal{A}_{t}^{\omega} g\right)=\lambda_{\omega}(t) \nu_{\omega}(t)(g)$ holds true for any bounded Borel function $g: \mathcal{E}_{\omega} \rightarrow \mathbb{C}$.

Moreover, this triplet is analytic and uniformly bounded around 0 . Namely, the maps

$$
\lambda_{\omega}(\cdot): U \rightarrow \mathbb{C}, h_{\omega}(\cdot): U \rightarrow \mathcal{H}_{\omega}^{\alpha, \xi} \text { and } \nu_{\omega}(\cdot): U \rightarrow\left(\mathcal{H}_{\omega}^{\alpha, \xi}\right)^{*}
$$

are analytic, where $\left(\mathcal{H}_{\omega}^{\alpha, \xi}\right)^{*}$ is the dual space of $\mathcal{H}_{\omega}^{\alpha, \xi}$, and for any $k \geq 0$ there is a constant $C_{k}>0$ so that

$$
\max \left(\sup _{z \in U}\left|\lambda_{\omega}^{(k)}(z)\right|, \sup _{z \in U}\left\|h_{\omega}^{(k)}(z)\right\|_{\alpha, \xi} \sup _{z \in U}\left\|\nu_{\omega}^{(k)}(z)\right\|_{\alpha, \xi}\right) \leq C_{k}, P \text {-a.s. }
$$

where $g^{(k)}$ stands for the $k$-th derivative of a function on the complex plane which takes values in some Banach space and $\|\nu\|_{\alpha, \xi}$ is the operator norm of a linear functional $\nu: \mathcal{H}_{\omega}^{\alpha, \xi} \rightarrow \mathbb{C}$.

Furthermore, there exist constants $m_{0}, C$ and $c \in(0,1)$ so that $P$-a.s. for any $z \in U, n \geq m_{0}$ and $q \in \mathcal{H}_{\omega}^{\alpha, \xi}$,

$$
\left\|\frac{\mathcal{A}_{z}^{\omega, n} q}{\lambda_{\omega, n}(z)}-\left(\nu_{\omega}(z) q\right) h_{\theta^{n} \omega}(z)\right\|_{\alpha, \xi} \leq C\|q\|_{\alpha, \xi} \cdot c^{n}
$$

where $\mathcal{A}_{z}^{\omega, n}=\mathcal{A}_{z}^{\theta^{n-1} \omega} \circ \mathcal{A}_{z}^{\theta^{n-2} \omega} \circ \cdots \circ \mathcal{A}_{z}^{\omega}$ and $\lambda_{\omega, n}(z)=\prod_{j=0}^{n-1} \lambda_{\theta^{j} \omega}(z)$.

Since $\mathcal{A}_{0}^{\omega} \mathbf{1}=\mathbf{1}$ we have $\lambda_{\omega}(0)=1$ and $h_{\omega}(0) \equiv \mathbf{1}$. Remark that we can always take

$$
\lambda_{\omega}(z)=\frac{a_{\omega}(z) \boldsymbol{\lambda}_{\omega}(z)}{a_{\theta \omega}(z) \boldsymbol{\lambda}_{\omega}(0)}, h_{\omega}(z)=\frac{a_{\omega}(z) \mathbf{h}_{\omega}(z)}{\mathbf{h}_{\omega}(0)} \text { and } \nu_{\omega}(z)=\left(a_{\omega}(z)\right)^{-1} \mathbf{h}_{\omega}(0) \boldsymbol{\nu}_{\omega}(z)
$$


where $\left(\boldsymbol{\lambda}_{\omega}(z), \mathbf{h}_{\omega}(z), \boldsymbol{\nu}_{\omega}(z)\right)$ is the RPF triplet corresponding to $\mathcal{L}_{z}^{\omega}$ and $a_{\omega}(z)=$ $\boldsymbol{\nu}_{\omega}(z)\left(\mathbf{h}_{\omega}(0)\right)$ (which is nonzero, see [13]). In particular, $\nu_{\omega}(0)=\boldsymbol{\mu}_{\omega}(0)$ and $\nu(0):=$ $\int \nu_{\omega}(0) d P(\omega)=\boldsymbol{\mu}(0)$.

In the special case when $e^{-\phi_{\omega}}$ is the appropriate Jacobian we have the following

3.2. Proposition. Let $\boldsymbol{m}$ be a probability measure on $\mathcal{X}$ and suppose $T_{\omega}$ is nonsingular with respect to $m, P$-a.s. Let $\phi_{\omega}=-\ln \left(\frac{d\left(T_{\omega}\right)_{*} m}{d m}\right)$ and assume that it is a Hölder continuous function and that $\left\|\phi_{\omega}\right\|_{\alpha, \xi}$ is a bounded random variable. Then $\boldsymbol{\nu}_{\omega}(0)=\boldsymbol{m}, \boldsymbol{\lambda}_{\omega}(0)=1$.

When $\phi_{\omega}=-\ln \left(\frac{d\left(T_{\omega}\right)_{*} m}{d m}\right)+\psi_{\omega}-\psi_{\theta \omega} \circ T_{\omega}+K_{\omega}$ for some bounded function $\psi_{\omega}(x)=\psi(\omega, x)$ and a random variable $K_{\omega}$, then $\nu_{\omega}(0)$ is equivalent to $\boldsymbol{m}$ and the Radon-Nykodim derivative is bounded from above and below by positive constants.

Proof. Suppose that $\phi_{\omega}=-\ln \left(\frac{d\left(T_{\omega}\right)_{*} \mathbf{m}}{d \mathbf{m}}\right)$. Then $\mathcal{L}_{0}^{\omega}$ is the dual operator of the (Koopman) operator $g \rightarrow g \circ T_{\omega}$ with respect to $\mathbf{m}$. In particular, $\mathbf{m}\left(\mathcal{L}_{0}^{\omega, n} \mathbf{1}\right)=1$ for any $n \geq 1$. Taking $\mu_{n}=\mathbf{m}$ in the limit expression of $\boldsymbol{\nu}_{\omega}(0)$ in (4.3.25) from [13. (see also Theorem 5.3.1 from there ) we obtain that for any Hölder continuous function $g: \mathcal{X} \rightarrow \mathbb{R}$,

$$
\int g d \boldsymbol{\nu}_{\omega}(0)=\lim _{n \rightarrow \infty} \frac{\mathbf{m}\left(\mathcal{L}_{0}^{\omega, n} g\right)}{\mathbf{m}\left(\mathcal{L}_{0}^{\omega, n} \mathbf{1}\right)}=\lim _{n \rightarrow \infty} \frac{\mathbf{m}(g)}{\mathbf{m}(\mathbf{1})}=\mathbf{m}(g)
$$

where in the first equality we used the duality relation discussed above. Since $\boldsymbol{\nu}_{\omega}(0)$ and $\mathbf{m}$ agree (as linear functionals) on $\mathcal{H}^{\alpha, \xi}$ we obtain that the measures $\boldsymbol{\nu}_{\omega}(0)$ and $\mathbf{m}$ are identical. Since $\boldsymbol{\lambda}_{\omega}(0)=\boldsymbol{\nu}_{\theta \omega}(0)\left(\mathcal{L}_{0}^{\omega} \mathbf{1}\right)$ and $\mathbf{m}\left(\mathcal{L}_{0}^{\omega} \mathbf{1}\right)=1$ we obtain from $\nu_{\omega}(0)=\mathbf{m}$ that $\boldsymbol{\lambda}_{\omega}(0)=1$.

The proof in the case when $\phi_{\omega}=-\ln \left(\frac{d\left(T_{\omega}\right)_{*} \mathbf{m}}{d \mathbf{m}}\right)+\psi_{\omega}-\psi_{\theta \omega} \circ T_{\omega}+K_{\omega}$ proceeds in a similar way since then $\mu\left(\mathcal{L}_{0}^{\omega, n} g\right)=e^{\sum_{j=0}^{n-1} K_{\theta^{j} \omega}} \mathbf{m}\left(g e^{\psi_{\omega}-\psi_{\theta^{n}} \circ T_{\omega}^{n}}\right)$.

The equalities $\boldsymbol{\nu}_{\omega}(0)=\mathbf{m}$ and $\boldsymbol{\lambda}_{\omega}(0)=1$ in Propositon 3.2 were stated in 23 in the case when $\mathcal{X}$ is a $C^{2}$-compact Riemanian manifold and $\mathbf{m}$ is the (normalized) volume measure (in fact, the proof comes from [20]). In [23] the random variable $\left\|\phi_{\omega}\right\|_{\alpha, \xi}$ is only assumed to be integrable. Under this integrability condition, the proof from [13] of existence of RPF trpilets proceeds similarly to [20] for real t's, so the condition that $\left\|\phi_{\omega}\right\|_{\alpha, \xi}$ is not really necessary in Proposition 3.2

3.1. Pressure near 0. Suppose that the assumptions of Theorem 3.1 hold true. We will gather several useful results concerning the behaviour of the logarithm of $\lambda_{\omega, n}(z)$ around 0 which were proved in Section 7.2 of [13]. The first one is

3.3. Lemma. There exists a neighborhood $U_{1}$ of 0 so that $P$-a.s. for any $k=1,2, \ldots$ there is an analytic function $\Pi_{\omega, k}: U_{1} \rightarrow \mathbb{C}$ such that

$$
e^{\Pi_{\omega, k}(z)}=\lambda_{\omega, k}(z) \text { and }\left|\Pi_{\omega, k}(z)\right| \leq k(\ln 2+\pi)
$$

for any $z \in U_{1}$.

The second is

3.4. Lemma. There exists a constant $Q_{2}>0$ so that $P$-a.s. for any $k \in \mathbb{N}$,

$$
\Pi_{\omega, k}^{\prime}(0)=\int S_{k}^{\omega} u d \nu_{\omega}(0) \quad \text { and }\left|\Pi_{\omega, k}^{\prime \prime}(0)-\operatorname{var}_{\nu_{\omega}(0)} S_{k}^{\omega} u\right| \leq Q_{2} .
$$


The third is

3.5. Lemma. Suppose that $\sigma^{2}>0$. Then there exist constants $t_{0}, c_{0}>0$ such that $P$-a.s. for any $z$ with $|z| \leq t_{0}$ and $k \geq 1$,

$$
\left|\Pi_{\omega, k}(z)-z \Pi_{\omega, k}^{\prime}(0)-\frac{z^{2}}{2} \Pi_{\omega, k}^{\prime \prime}(0)\right| \leq c_{0}|z|^{3} k
$$

3.2. Additional estimates. We gather here several estimates derived in [13] which will be in constant use in the course of the proofs of the results stated in Section 2. We begin with the following random Lasota-Yorke type inequality (see Lemma 5.6.1 in [13): there exists a constant $Q>0$ so that $P$-a.s. for any $n \geq 1$, $z \in \mathbb{C}$ and $g \in \mathcal{H}^{\alpha, \xi}$,

$$
\begin{array}{r}
\left\|\mathcal{L}_{z}^{\omega, n} g\right\|_{\alpha, \xi} \leq\left\|\mathcal{L}_{0}^{\omega, n} \mathbf{1}\right\|_{\infty} e^{|\Re(z)|\left\|S_{n}^{\omega} u\right\|_{\infty}} \\
\times\left(v_{\alpha, \xi}(g)\left(\gamma_{\omega, n}\right)^{-\alpha}+(1+2 Q)\left(1+\|z\|_{1}\right)\|g\|_{\infty}\right) .
\end{array}
$$

where $\|z\|_{1}=|\Re(z)|+|\Im(z)|$ and $\Re(z)(\Im(z))$ is the real (imaginary) part of $z$. In particular, $\mathcal{L}_{z}^{\omega, n}\left(\mathcal{H}_{\omega}^{\alpha, \xi}\right) \subset \mathcal{H}_{\theta^{n} \omega}^{\alpha, \xi}$ and the corresponding operator norms satisfy

$$
\left\|\mathcal{L}_{i t}^{\omega, n}\right\|_{\alpha, \xi} \leq B(1+|t|), t \in \mathbb{R}
$$

where $B$ is some constant. Next, similarly to proof of Corollary 5.12.3 in [13, there exists a constant $B_{0}>1$ so that $P$-a.s. for any $n \geq 1$,

$$
\left\|\mathcal{L}_{z}^{\omega, n}\right\|_{\alpha, \xi} B_{0}^{-1} \leq\left\|\mathcal{A}_{z}^{\omega, n}\right\|_{\alpha, \xi} \leq B_{0}\left\|\mathcal{L}_{z}^{\omega, n}\right\|_{\alpha, \xi}
$$

and therefore $\left\|\mathcal{A}_{i t}^{\omega, n}\right\|_{\alpha, \xi} \leq B_{2}(1+|t|)$ where $B_{2}=B B_{0}$.

Finally, since $\lambda_{\omega, n}(z)=\nu_{\omega}(z)\left(\mathcal{A}_{z}^{\omega, n} \mathbf{1}\right)$, applying (3.2) and (3.10) yields that there exists a constant $A$ so that $P$-a.s.,

$$
\left|\lambda_{\omega, n}(i t)\right| \leq A\left\|\mathcal{A}_{i t}^{\omega, n}\right\|_{\alpha, \xi} \leq A B_{2}
$$

for any $n \geq 1$ and real $t$ so that it $\in U$. Note that in the integral operator case it is trivial that $\left\|R_{i t}^{\omega, n}\right\|_{\infty} \leq 1$, so there is no need in using additional results in order to obtain appropriate upper bounds on the latter norms.

\section{Annealed limit theorems}

4.1. Reduction to the invertible case. Let $(\Omega, \mathcal{F}, P, \theta)$ be a measure preserving system and let $T_{\omega}, \phi_{\omega}$ and $u_{\omega}$ be as described at the beginning of Section 2 Recall the following definition. We say that $(\hat{\Omega}, \hat{\mathcal{F}}, \hat{P}, \hat{\theta})$ is the natural (invertible) extension of $(\Omega, \mathcal{F}, P, \theta)$ if $\hat{\Omega} \subset \Omega_{0}^{\mathbb{Z}}$ is the space of all two sided sequences $\hat{\zeta}=\left(\ldots \zeta_{-1}, \zeta_{0}, \zeta_{1} \ldots\right)$ so that $\theta \zeta_{i}=\zeta_{i+1}$ for any $i, \hat{\theta}$ is the shift map defined by $(\hat{\theta} \zeta)_{i}=\zeta_{i+1}, \hat{\mathcal{F}}$ is the $\theta$-algebra induced on $\Omega$ by the product $\sigma$-algebra $\mathcal{F}^{\mathbb{Z}}$ and $\hat{P}$ is the probability measure defined by $\hat{P}\left\{\hat{\zeta}: \zeta_{i} \in A_{i} ;|i| \leq M\right\}=P_{0}\left(\bigcap_{i=0}^{2 M} \theta^{-i} A_{i-M}\right)$. When $\Omega$ is a metric space and $\mathcal{F}$ contains its Borel $\sigma$-algebra then $\hat{\Omega}$ is a metric space and $\hat{\mathcal{F}}$ contains the appropriate Borel $\sigma$-algebra. Set $\mathcal{T}_{\hat{\zeta}}=T_{\zeta_{0}}$ and consider the skew product map $\mathcal{T}$ given by $\mathcal{T}(\hat{\zeta}, x)=\left(\hat{\theta} \hat{\zeta}, T_{\hat{\zeta}} x\right)$. Let $\pi_{0}: \hat{\Omega} \rightarrow \Omega$ be the projection on the 0 -th coordinate given by $\pi_{0} \hat{\zeta}=\zeta_{0}$. Then $\pi_{0}$ is a factor map. Set $\pi(\hat{\zeta}, x)=\left(\pi_{0} \hat{\zeta}, x\right)=\left(\zeta_{0}, x\right)$ and let $\boldsymbol{\mu}=P \times \kappa$ be the measure described in Section 2.2. Then $\hat{\boldsymbol{\mu}}:=\pi_{*} \boldsymbol{\mu}$ is the Gibbs measure described before Theorem 2.4, i.e. the measure whose disintegrations are given by $\boldsymbol{\mu}_{\hat{\zeta}}=\boldsymbol{h}_{\hat{\zeta}}(0) \boldsymbol{\nu}_{\hat{\zeta}}(0)$. Indeed, in our circumstances Proposition 3.2 shows that $\boldsymbol{\nu}_{\hat{\zeta}}(0)=\mathbf{m}$ and that $\boldsymbol{\lambda}_{\hat{\zeta}}(0)=1$. 
Therefore, it will be sufficient to show that $\boldsymbol{h}_{\hat{\zeta}}=\bar{h}, \hat{P}$-a.s.. For this purpose, for any continuous function $g$ on $\mathcal{X}$ write

$$
\kappa(g)=\mathbf{m}(\bar{h} g)=\mathbf{m}\left(\bar{h} \cdot g \circ T_{\zeta_{0}}\right)=\boldsymbol{\nu}_{\hat{\zeta}}(0)\left(\bar{h} \cdot g \circ \mathcal{T}_{\hat{\zeta}}\right)=\boldsymbol{\nu}_{\hat{\theta} \hat{\zeta}}(0)\left(\mathcal{L}_{0}^{\hat{\zeta}} \bar{h} \cdot g\right)=\mathbf{m}\left(\mathcal{L}_{0}^{\hat{\zeta}} \bar{h} \cdot g\right)
$$

where in the second equality we used the $T$-invariance of $\boldsymbol{\mu}=P \times \kappa$. Since these equalities hold true for any continuous $g$ and $\bar{h}$ is continuous, we derive that $\mathcal{L}_{0}^{\hat{\zeta}} \bar{h}=$ $\bar{h}$. Now, as in the proof of Propostion 3.19 in [24], we derive from (3.3) that the left hand side of (3.3) converges to 0 as $n \rightarrow \infty$ for any continuous function $q: \mathcal{X} \rightarrow \mathbb{C}$. Since $\bar{h}$ is a density function we have $\mathbf{m}(\bar{h})=1$, and therefore, taking $q=\bar{h}$ yields that the sequence $\boldsymbol{h}_{\hat{\theta}^{n} \hat{\zeta}}(0)$ converges to $\bar{h}$. We claim next that $\boldsymbol{h}_{\hat{\zeta}}=\bar{h}, \hat{P}$-a.s. Indeed, for any $x \in \mathcal{X}$ we have

$$
0=\lim _{n \rightarrow \infty} \frac{1}{n} \sum_{j=0}^{n-1}\left|\boldsymbol{h}_{\hat{\theta} j \hat{\zeta}}(0)(x)-\bar{h}(x)\right|, \hat{P}-a . s .
$$

implying that $\int\left|\boldsymbol{h}_{\zeta}(0)(x)-\bar{h}(x)\right| d \hat{P}(\hat{\zeta})=0$, or, equivalently, that $\boldsymbol{h}_{\zeta}(0)(x)=$ $\bar{h}(x)=0, \hat{P}$-a.s. By compactness of $\mathcal{X}$ and continuity of both $\boldsymbol{h}_{\zeta}(0)$ and $\bar{h}$ we obtain the desired equality.

Set $U_{\hat{\zeta}}=u_{\zeta_{0}}$, and $\Phi_{\hat{\zeta}}=\phi_{\zeta_{0}}$. Then $\hat{\boldsymbol{\mu}}_{\hat{\zeta}}\left(U_{\hat{\zeta}}\right)=\kappa\left(u_{\zeta_{0}}\right)=\boldsymbol{\mu}_{\zeta_{0}}\left(u_{\zeta_{0}}\right)$ and the distribution of the processes $\left\{\left(U \circ \mathcal{T}^{n}\right)(\hat{\zeta}, x): n \geq 0\right\}$ and $\left\{\left(u \circ T^{n}\right)(\omega, y): n \geq 0\right\}$ are the same when $(\hat{\zeta}, x)$ is distributed according to $\hat{\boldsymbol{\mu}}$ and $(\omega, y)$ is distributed according to $\boldsymbol{\mu}$. Finally, the periodic points of $\theta$ are exactly the points of the form $\hat{\zeta}=(\ldots a, a, a, \ldots)$, where $a=\left(\omega_{0}, \theta \omega_{0}, \ldots, \theta^{n_{0}-1} \omega_{0}\right)$ for some periodic point $\omega_{0}$ of $\theta$ whose period is $n_{0}$. Therefore, all the conditions in Theorems 2.4, 2.7 and 2.8 hold true with $\mathcal{T}_{\hat{\zeta}}, \Phi_{\hat{\zeta}}$ and $U_{\hat{\zeta}}$ when they hold with $T_{\omega}, \phi_{\omega}$ and $u_{\omega}$.

4.1. Remark. Applying the results stated in Section 2.1, we obtain limit theorems in the non-invertible case also when $T_{\omega}$ 's do not necessarily preserve the absolutely continuous measure. Indeed, let $\hat{\boldsymbol{\mu}}$ be the Gibbs measure (in the extension) and set $\boldsymbol{\mu}=\pi_{*} \hat{\boldsymbol{\mu}}$. Then $\boldsymbol{\mu}$ is $T$-invariant and the distribution of the processes $\{(U \circ$ $\left.\left.\mathcal{T}^{n}\right)(\hat{\zeta}, x): n \geq 0\right\}$ and $\left\{\left(u \circ T^{n}\right)(\omega, y): n \geq 0\right\}$ are the same when $(\hat{\zeta}, x)$ is distributed according to $\hat{\boldsymbol{\mu}}$ and $(\omega, y)$ is distributed according to $\boldsymbol{\mu}$. The problem here is that the condition that $\hat{\boldsymbol{\mu}}_{\hat{\zeta}}\left(U_{\hat{\zeta}}\right)$ does not depend on $\hat{\zeta}$ can not be easily expressed in terms of the original non-invertible system $(\Omega, \mathcal{F}, P, \theta)$, as explained in the following. The limit expressions (4.3.25) and (4.3.28) in Chapter 4 of [13] show that $\boldsymbol{\lambda}_{\hat{\zeta}}(z)$ and $\boldsymbol{\nu}_{\hat{\zeta}}(z)$ depend only on $\zeta_{0}$, but in general, the function $\boldsymbol{h}_{\hat{\zeta}}(z)$ does not depend only on $\zeta_{0}$ since (see (3.3)),

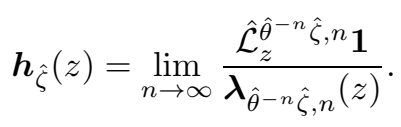

Therefore, the Gibbs measure $\boldsymbol{\mu}_{\hat{\zeta}}(0)$ does not depend only on $\hat{\zeta}_{0}$. Since $\hat{\theta}$ is ergodic, the condition that $\boldsymbol{\mu}_{\hat{\zeta}}(0)\left(U_{\hat{\zeta}}\right)$ does not depend on $\hat{\zeta}$ is equivalent to existence of the $\operatorname{limit}_{n \rightarrow \infty} \lim _{n \rightarrow \hat{\theta^{n}} \hat{\zeta}}(0)\left(U_{\hat{\theta}^{n} \hat{\zeta}}\right), \hat{P}$-a.s., which together with (3.3) , is equivalent to existence of the limits of

$$
\left(\boldsymbol{\lambda}_{\hat{\zeta}, n}(0)\right)^{-1} \boldsymbol{\nu}_{\hat{\theta}^{n} \hat{\zeta}}(0)\left(U_{\hat{\theta}^{n} \hat{\zeta}} \hat{\mathcal{L}}_{0}^{\hat{\zeta}, n} \mathbf{1}\right)=\boldsymbol{\nu}_{\hat{\zeta}}(0)\left(U_{\hat{\theta}^{n} \hat{\zeta}^{\circ}} \circ T_{\hat{\zeta}}^{n}\right)
$$


as $n \rightarrow \infty$ (note that the latter expressions depend only on $\zeta_{0}$ ). By proposition 3.2, when $\phi_{\omega}=-\ln \left(\frac{d\left(T_{\omega}\right)_{*} \mathbf{m}}{d \mathbf{m}}\right)$ then $\boldsymbol{\nu}_{\hat{\zeta}}(0)=\mathbf{m}$, and so in this case the condition that $\boldsymbol{\mu}_{\hat{\zeta}}(0)$ does not depend on $\hat{\zeta}$ is equivalent to convergence of $\mathbf{m}\left(u_{\theta^{n} \omega} \circ T_{\omega}^{n}\right)$ to $\boldsymbol{\mu}(u)=\int u d \boldsymbol{\mu}$.

4.2. Characteristic functions and Markov chains. We begin with the following. Let $\xi_{n}^{\theta^{-n} \omega}, n \geq 0$ be the Markov chain generated by the initial distribution $\boldsymbol{\mu}_{\omega}=\nu_{\omega}(0)$ on $\mathcal{X}$ and the $n$-th step transition operators $\mathcal{A}_{0}^{\theta^{-n} \omega, n}$, where $\mathcal{A}_{z}^{\omega, j}=\mathcal{A}_{z}^{\theta^{j-1} \omega} \circ \mathcal{A}_{z}^{\theta^{j-2} \omega} \circ \cdots \circ \mathcal{A}_{z}^{\omega}$ for any complex $z$ and natural $j$. Set $S_{n}=\sum_{j=0}^{n-1} u \circ T^{n}$,

$$
\mathcal{S}_{n}^{\omega}=\sum_{j=0}^{n-1} u_{\theta^{-j} \omega}\left(\xi_{j}^{\theta^{-j} \omega}\right)
$$

and let $\psi_{\omega, n}(t)$ be the characteristic function of $\mathcal{S}_{n}^{\omega}$. When $x$ is distributed according to $\boldsymbol{\mu}_{\omega}:=\boldsymbol{\mu}_{\omega}(0)$ we have

$$
\left(x, T_{\omega} x, T_{\omega}^{2} x, \ldots, T_{\omega}^{n} x\right) \stackrel{d}{=}\left(\xi_{n}^{\theta^{\omega}}, \xi_{n-1}^{\theta^{n} \omega}, \ldots, \xi_{0}^{\theta^{n} \omega}\right)
$$

where $\stackrel{d}{=}$ stands for equality in distribution. Therefore,

$$
\mathbb{E} e^{i t S_{n}}=\int \psi_{\omega, n}(t) d P(\omega)=\int \psi_{\theta^{n} \omega, n}(t) d P(\omega)=\int \boldsymbol{\mu}_{\theta^{n} \omega}\left(\mathcal{A}_{i t}^{\omega, n} \mathbf{1}\right) d P(\omega) .
$$

4.3. Proof of the CLT. We assume here that $\nu_{\omega}(0)\left(u_{\omega}\right)=0$. Set $\hat{S}_{n}=n^{-\frac{1}{2}} S_{n}$. By the Levi continuity theorem, it suffice to show that there exists $r>0$ so that for any $t \in[-r, r]$,

$$
\lim _{n \rightarrow \infty} \mathbb{E} e^{i t \hat{S}_{n}}=e^{-\frac{1}{2} \sigma^{2} t^{2}} .
$$

In order to prove the latter equality, we first write

$$
\mathbb{E} e^{i t \hat{S}_{n}}=\int \boldsymbol{\mu}_{\theta^{n} \omega}\left(\mathcal{A}_{i t_{n}}^{\omega, n} \mathbf{1}\right) d P(\omega)
$$

where $t_{n}=n^{-\frac{1}{2}} t$. Set

$$
\varphi_{\omega, n}(z)=\int \frac{\mathcal{A}_{z}^{\omega, n} \mathbf{1}}{\lambda_{\omega, n}(z)} d \boldsymbol{\mu}_{\theta^{n} \omega}
$$

Then we can write

$$
\mathbb{E} e^{i t \hat{S}_{n}}=\int e^{\Pi_{\omega, n}\left(i t_{n}\right)} \varphi_{\omega, n}\left(i t_{n}\right) d P(\omega)
$$

where $\Pi_{\omega, n}$ is defined in Lemma 3.3. As in the proof of Theorem 7.1.1 in [13, there exist constants $B, b>0$ such that for any $n \geq 1$ and $z$ so that $|z| \leq b$,

$$
\left|\varphi_{\omega, n}(z)-\varphi_{\omega, n}(0)\right|=\left|\varphi_{\omega, n}(z)-1\right| \leq B|z|^{2} .
$$

Let $\varepsilon \in(0,1)$ and $N_{\varepsilon}$ be so that for any $n \geq N_{\varepsilon}$ we have $P\left(\Gamma_{n, \varepsilon}\right) \geq 1-\varepsilon$, where

$$
\Gamma_{n, \varepsilon}=\left\{\omega:\left|n^{-1} \operatorname{var}_{\nu_{\omega}(0)} S_{n}^{\omega} u-\sigma^{2}\right| \leq \varepsilon\right\} .
$$

Then for any $n \geq N_{\varepsilon}$ and $t \in[-b, b]$,

$$
\left|\mathbb{E} e^{i t \hat{S}_{n}}-e^{-\frac{1}{2} \sigma^{2} t^{2}}\right| \leq 2 C \varepsilon+\int_{\Gamma_{n, \varepsilon}}\left|e^{\Pi_{\omega, n}\left(i t_{n}\right)} \varphi_{\omega, n}\left(i t_{n}\right)-e^{-\frac{1}{2} \sigma^{2} t^{2}}\right| d P(\omega)
$$


where $C>0$ is some constant, and we used (3.11) and that $e^{\Re \bar{\Pi}_{\omega, n}(i t)}=\left|\lambda_{\omega, n}(i t)\right|$. Next, by lemma 3.4. Lemma 3.5. (3.11) and (4.4) there exists a constant $D>0$ so that for any $t \in[-b, b], \varepsilon>0, n \geq N_{\varepsilon}$ and $\omega \in \Gamma_{n, \varepsilon}$,

$$
\begin{array}{r}
\left|e^{\Pi_{\omega, n}\left(i t_{n}\right)} \varphi_{\omega, n}\left(i t_{n}\right)-e^{-\frac{1}{2} \sigma^{2} t^{2}}\right| \leq e^{\Re \bar{\Pi}_{\omega, n}\left(i t_{n}\right)}\left|\varphi_{\omega, n}\left(i t_{n}\right)-1\right| \\
+\left|e^{\Pi_{\omega, n}\left(i t_{n}\right)}-e^{-\frac{1}{2} \sigma^{2} t^{2}}\right| \leq D\left(n^{-1}+n^{-\frac{1}{2}}+e^{\varepsilon}-1\right) .
\end{array}
$$

Equality (4.3) follows now by taking $n \rightarrow \infty$ and then $\varepsilon \rightarrow 0$.

4.4. Norms estimates for small t's. For $P$ a.a. $\omega$ and $n \in \mathbb{N}$, write

$$
\left|\lambda_{\omega, n}(i t)\right|=e^{\Re \Pi_{\omega, n}(i t)} .
$$

By Lemmas 3.4 and 3.5 we derive that for any $c>0$ there exist positive constants $\delta_{0}$ and $c_{1}$ so that for any $t \in\left[-\delta_{0}, \delta_{0}\right]$ and $n \geq 1$ such that $\operatorname{var}_{\nu_{\omega}(0)} S_{n}^{\omega} u \geq c n$,

$$
\left|\lambda_{\omega, n}(i t)\right| \leq A e^{-c_{1} n t^{2}}
$$

Therefore, under Assumption 2.6, there exist positive constants $d_{1}, d_{2}$ and $\delta_{0}$ and sets $\Gamma_{n}, n \in \mathbb{N}$ so that

$$
1-P\left(\Gamma_{n}\right) \leq \frac{d_{1}}{n^{\beta}}
$$

and for any $\omega \in \Gamma_{n}$ and $t \in\left[-\delta_{0}, \delta_{0}\right]$,

$$
\left|\lambda_{\omega, n}(i t)\right| \leq A e^{-d_{2} n t^{2}}
$$

Note that by (3.2) and (3.3), there exists a constant $A^{\prime}$ so that

$$
\left\|\mathcal{A}_{i t}^{\omega, n}\right\|_{\alpha, \xi} \leq A^{\prime}\left|\lambda_{\omega, n}(i t)\right|
$$

and so we obtain on $\Gamma_{n}$ appropriate estimates of $\left\|\mathcal{A}_{i t}^{\omega, n}\right\|_{\alpha, \xi}$ and $\left\|\mathcal{L}_{i t}^{\omega, n}\right\|_{\alpha, \xi}$, as well.

4.2. Remark. Suppose that $\sigma^{2}>0$ and set $V_{n}(\omega)=\operatorname{var}_{\nu_{\omega}(0)} S_{n}^{\omega} u$. In Chapter 7 of 13 we proved that, $P$-a.s., the converges rate of of law of $\left(V_{n}(\omega)\right)^{-\frac{1}{2}} S_{n}^{\omega} \bar{u}$ towards the standard normal law is optimal. When Assumption 2.6 hold true with $\beta=\frac{1}{2}$, then (4.7) and the arguments in 13] yield the following result: there exists a constant $c>0$ so that for any $n \geq 1$,

$$
\sup _{r \in \mathbb{R}}\left|\boldsymbol{\mu}\left\{(\omega, x): S_{n}^{\omega} \bar{u}_{\omega}(x) \leq r \sqrt{V_{n}(\omega)}\right\}-\frac{1}{\sqrt{2 \pi}} \int_{-\infty}^{r} e^{-\frac{1}{2} t^{2}} d t\right| \leq c n^{-\frac{1}{2}} .
$$

This is not the Berry-Essen theorem for the self normalized sums $\left(\operatorname{var}_{\mu} S_{n} \bar{u}\right)^{-\frac{1}{2}} S_{n} \bar{u}$. Obtaining estimates on the left hand side of (4.8) with $\operatorname{var}_{\mu} S_{n} \bar{u}=\mathbb{E}_{P} V_{n}(\omega)$ in place of $V_{n}(\omega)$ requires concentration inequalities of the form

$$
P\left\{\omega:\left|V_{n}(\omega)-\mathbb{E}_{P} V_{n}(\omega)\right| \geq c_{1} n^{\delta_{1}}\right\} \leq c_{2} n^{-\delta_{2}}
$$

for some $c_{1}, c_{2}, \delta_{1}, \delta_{2}>0$. When $\delta_{1}=\delta_{2} \geq \frac{1}{2}$ this would yield the rate $n^{-\frac{1}{2}}$, while in general we would get a (possibly) smaller negative power of $n$. The problem here is that such inequalities do not seem to hold true under general conditions, as demonstrated in the following. Let $\theta$ be a Young tower. When $\boldsymbol{\mu}_{\omega}=\mu$ and $\boldsymbol{\mu}_{\omega}\left(u_{\omega}\right)=\gamma$ do not depend on $\omega$, set $\hat{V}_{n}\left(\omega_{0}, \ldots, \omega_{n-1}\right)=\operatorname{var}_{\mu}\left(\sum_{j=0}^{n-1} u_{\omega_{0}} \circ T_{\omega_{j}} \circ T_{\omega_{j-1}} \circ\right.$ $\left.\cdots \circ T_{\omega_{0}}\right)$. The function $\hat{V}_{n}$ is Hölder continuous when the family $\left\{T_{\omega}(\cdot): \omega \in \Omega\right\}$ is uniformly Hölder continuous, and $u_{\omega}$ and $T_{\omega}$ are Hölder continuous functions of the variable $\omega$. The Hölder constant of $\hat{V}_{n}$ at direction $\omega_{0}$ grows exponentially fast in $n$, which makes it impossible to apply effectively the concentration inequalities 
of the form used in [5] (see Section [5), or any other reasonable general type of concentration inequality.

4.5. Norms estimates for large t's. We will prove here the following

4.3. Lemma. Suppose that (2.8) from Assumption 2.6 holds true. In the non-lattice case, let $J \subset \mathbb{R}$ be a compact set not containing the origin. In the lattice case, let $J$ be a compact subset of $\left(-\frac{2 \pi}{h}, \frac{2 \pi}{h}\right) \backslash\{0\}$. Then there exist sets $\Delta_{n}=\Delta_{n}(J), n \geq 1$ and positive constants $d=d(J)$ and $u=u(J)$ so that for any $n \geq 1$,

$$
1-P\left(\Delta_{n}\right) \leq \frac{d}{n^{\beta}}
$$

and for any $\omega \in \Delta_{n}$,

$$
\sup _{t \in J}\left\|\mathcal{A}_{i t}^{\omega, n}\right\|_{\alpha, \xi} \leq 4 B_{0} \cdot 2^{-u n}
$$

where $B_{0}$ comes from (3.10).

Proof. First, by (3.9) there exists a constant $B>0$ so that $P$-a.s. for any real $t$,

$$
\left\|\mathcal{L}_{i t}^{\omega, n}\right\|_{\alpha, \xi} \leq B(1+|t|):=B_{t} .
$$

Consider the transfer operators $R_{i t}, t \in \mathbb{R}$ generated by the function $S_{n_{0}}^{\omega_{0}} u$ and the map $\tau=T_{\omega_{0}}^{n_{0}}$, namely

$$
R_{i t}=\mathcal{L}_{i t}^{\omega_{0}, n_{0}}
$$

Observe that for each $t$ the spectral radius of $R_{i t}$ does not exceed 1 since the norms $\left\|\mathcal{L}_{i t}^{\omega, n}\right\|_{\alpha, \xi}$ are bounded in $\omega$ and $n$. In the non-lattice case, let $J \subset \mathbb{R}$ be a compact set not containing 0 , while in the lattice case let $J$ be a compact subset of $\left(-\frac{2 \pi}{h}, \frac{2 \pi}{h}\right) \backslash\{0\}$. Then, in both cases, there there exist constants $A>0$ and $a \in(0,1)$, which may depend on $J$, so that for any $n \in \mathbb{N}$ and $t \in J$,

$$
\left\|R_{i t}^{n}\right\|_{\alpha, \xi} \leq A a^{n} \text {. }
$$

The above follows from classical spectral analysis type results together with our assumptions about the $R_{i t}$ 's, and we refer the readers' to [15] for the details. Let $J$ be a compact set as described above and set $B_{J}=\sup _{t \in J} B_{t}$. Let $s=s(J)$ be sufficiently large so that $A a^{s}<\frac{1}{4 B_{J}}$, where $A$ and $a$ satisfy (4.12). Since Assumption 2.5 holds true there exist neighborhoods $B_{\theta^{j} \omega_{0}}, 0 \leq j<n_{0}$ of the points $\theta^{j} \omega_{0}, 0 \leq$ $j<n_{0}$, respectively, so that

$$
\sup _{t \in J}\left\|\mathcal{L}_{i t}^{\omega, n_{0} s}-R_{i t}^{s}\right\|_{\alpha, \xi} \leq \frac{1}{4 B_{J}}
$$

for any $\omega \in B_{\omega_{0}, n_{0}, s}:=\bigcap_{i=0}^{s n_{0}-1} \theta^{-i} B_{\theta^{i} \omega_{0}}$, where we also used that $R_{i t}^{s}=\mathcal{L}_{i t}^{\omega_{0}, s n_{0}}$. It follows that for any $\omega \in B_{\omega_{0}, n_{0}, s}$,

$$
\sup _{t \in J}\left\|\mathcal{L}_{i t}^{\omega, n_{0} s}\right\|_{\alpha, \xi} \leq \frac{1}{2 B_{J}}
$$

Set

$$
\Delta_{n}=\left\{\omega \in \Omega: \sum_{j=0}^{n-1} \mathbb{I}_{B_{\omega_{0}, n_{0}, s}}\left(\theta^{j} \omega\right)>c n\right\}
$$

where $c$ comes from (2.6). Then

$$
1-P\left(\Delta_{n}\right) \leq \frac{d}{n^{\beta}}
$$


We also set

$$
\mathbf{B}_{\omega_{0}, n_{0}, s}=\left\{\left(u_{i}\right) \in \Omega^{s n_{0}}: u_{i} \in B_{\theta^{i} \omega_{0}} \forall 1 \leq i \leq s n_{0}\right\} .
$$

Then $\omega^{\prime} \in B_{\omega_{0}, n_{0}, s}$ if and only if the "word" $\left(\omega^{\prime}, \theta \omega^{\prime}, \ldots, \theta^{s n_{0}-1} \omega^{\prime}\right)$ belongs to $\mathbf{B}_{\omega_{0}, n_{0}, s}$. When $\omega \in \Delta_{n}$ the word $\left(\omega, \theta \omega, \ldots, \theta^{n-1} \omega\right)$ contains at least $\left[\frac{n c}{n_{0} s}\right]-1$ disjoint sub-words

$$
\omega\left(q, s n_{0}\right)=\left(\theta^{q+1} \omega, \ldots, \theta^{q+s n_{0}} \omega\right)
$$

which are contained in $\mathbf{B}_{\omega_{0}, n_{0}, s}$. Namely, there exist indexes $q_{1}, q_{2}, \ldots, q_{L}, L \geq$ $\left[\frac{n c}{n_{0} s}\right]-1$ so that each $\omega\left(q_{i}, s n_{0}\right)$ is a member of $\mathbf{B}_{\omega_{0}, n_{0}, s}$ and $q_{i}+s n_{0}<q_{i+1}$ for all $i=1,2, \ldots, L-1$. As a consequence, on $\Delta_{n}$ we can write

$$
\mathcal{L}_{i t}^{\omega, n}=\mathcal{B}_{t, 1}^{\omega} \circ \mathcal{C}_{t, 1}^{\omega} \circ \mathcal{B}_{t, 2}^{\omega} \circ \mathcal{C}_{t, j}^{\omega} \circ \cdots \circ \mathcal{C}_{t, L}^{\omega} \circ \mathcal{B}_{t, L+1}^{\omega}
$$

where $L=L_{\omega, n} \geq\left[\frac{c n}{n_{0} s}\right]-1$, each $\mathcal{C}_{t, j}^{\omega}$ satisfies

$$
\sup _{t \in J}\left\|\mathcal{C}_{t, j}^{\omega}\right\|_{\alpha, \xi} \leq \frac{1}{2 B_{J}}
$$

while each $\mathcal{B}_{t, j}^{\omega}$ satisfies

$$
\sup _{t \in J}\left\|\mathcal{B}_{t, j}^{\omega}\right\|_{\alpha, \xi} \leq B_{J}
$$

We conclude from the above estimates that on $\Delta_{n}$ we have

$$
\sup _{t \in J}\left\|\mathcal{A}_{i t}^{\omega, n}\right\|_{\alpha, \xi} \leq B_{0} \sup _{t \in J}\left\|\mathcal{L}_{i t}^{\omega, n}\right\|_{\alpha, \xi} \leq 4 B_{0} \cdot 2^{-u n}
$$

where $u=\frac{c}{n_{0} s}$, and we also used (3.10).

4.6. Proof of the LLT. By Theorem 2.4 and exactly as in the proof of Theorem 2.3 in [13, it is sufficient to show that Assumptions 2.2.1 and 2.2.2 from [13] hold true. These assumptions are verified using the inequality

$$
\begin{array}{r}
\left|\mathbb{E} e^{i t S_{n}}\right|=\left|\int \mu_{\omega}\left(\mathcal{A}_{i t}^{\theta^{-n} \omega, n} \mathbf{1}\right) d P(\omega)\right|=\left|\int \mu_{\theta^{n} \omega}\left(\mathcal{A}_{i t}^{\omega, n} \mathbf{1}\right) d P(\omega)\right| \\
\leq 1-P(\Gamma)+\left\|\mathbb{I}_{\Gamma}(\omega)\right\| \mathcal{A}_{i t}^{\omega, n}\left\|_{\alpha, \xi}\right\|_{L^{\infty}(\Omega, \mathcal{F}, P)}
\end{array}
$$

applied with either $\Gamma=\Gamma_{n}$ defined before (4.6) or with $\Gamma=\Delta_{n}$ from Lemma 4.3, taking into account that $\beta>\frac{1}{2}$.

4.7. Proof of the renewal theorem. In this section we will always assume that the conditions of Theorem 2.8 hold true. In the course of the proof, when it is more convenient, will use the notation $\mathbb{E}_{P}$ to denote integration over $\Omega$ with respect to $P$. Let $\mathscr{H}_{1}$ be the space of all continuous and integrable functions $g: \mathbb{R} \rightarrow \mathbb{C}$ whose Fourier transforms are continuously differentiable with compact support. Then by Lemma IV.5 in [15, it is sufficient in (2.9) from Theorem 2.8 to consider only functions $g \in \mathscr{H}_{1}$ which are dominated by some positive element of $\mathscr{H}_{1}$. Note that such functions satisfy the Fourier inversion formula. For any $\rho \in(0,1)$ set

$$
\begin{aligned}
U_{\rho}(B)= & \sum_{n \geq 1} \rho^{n-1} \int \mu_{\omega}\left(f_{\theta^{-n} \omega}\left(\xi_{n}^{\theta^{-n} \omega}\right) \mathbb{I}_{B}\left(S_{n}^{\omega}\right)\right) d P(\omega) \\
& =\sum_{n \geq 1} \rho^{n-1} \int \mathbb{E}_{\mu_{\theta^{n}}}\left[f_{\omega}\left(\xi_{n}^{\omega}\right) \mathbb{I}_{B}\left(S_{n}^{\theta^{n} \omega}\right)\right] d P(\omega) .
\end{aligned}
$$


Since $\mathbb{E}_{P}\left\|f_{\omega}\right\|_{\infty}<\infty$, it is clear that $U_{\rho}$ is a finite measure for each $\rho \in(0,1)$. Let $g$ a function of the described above form and suppose that its Fourier transform $\hat{g}$ vanishes outside the compact interval $[-b, b]$. In the non-lattice case set

$$
V_{\rho}(g)=\frac{1}{2 \pi} \int_{-\delta_{0}}^{\delta_{0}} \hat{g}(t) \mathbb{E}_{P}\left[\nu_{\omega}(i t)\left(f_{\omega}\right) f_{\rho}^{\omega}(t)\right] d t
$$

where

$$
f_{\rho}^{\omega}(t)=\sum_{n \geq 1} \rho^{n-1} \lambda_{\omega, n}(i t) \mu_{\theta^{n} \omega}\left(h_{\theta^{n} \omega}(i t)\right)
$$

which by (3.2) and (3.11) converges uniformly over $\omega$ and $t \in\left[-\delta_{0}, \delta_{0}\right]$. In the lattice case we set

$$
V_{\rho}(g)=\frac{1}{2 \pi} \int_{-\delta_{0}}^{\delta_{0}} r(t) \mathbb{E}_{P}\left[\nu_{\omega}(i t)\left(f_{\omega}\right) f_{\rho}^{\omega}(t)\right] d t
$$

where $r(t)=\sum_{k \in \mathbb{Z}} \hat{g}\left(t+\frac{2 \pi k}{h}\right)$. Note that by the so-called Poisson summation formula (see Ch. 10 in [4]) we have $r(0)=\sum_{k} \hat{g}\left(\frac{2 \pi k}{h}\right)=\int g d \kappa_{h}=\kappa_{h}(g)$.

4.4. Lemma. In both the lattice and non-lattice cases, there exist integrable functions $R_{1}$ and $R_{2}$ on $\mathbb{R}$ so that

$$
\lim _{\rho \rightarrow 1}\left(U_{\rho}(g)-V_{\rho}(g)\right)=\int_{\left\{t:|t|>\delta_{0}\right\}} e(t) R_{1}(t) d t+\int_{-\delta_{0}}^{\delta_{0}} e(t) R_{2}(t) d t
$$

where in the non-lattice case $e(t)=\hat{g}(t)$ while in the lattice case $e(t)=r(t)$.

Proof. For any $0<\rho<1$ set

$$
L_{\rho}(t)=\sum_{n \geq 1} \rho^{n-1} \mathbb{E}_{P}\left[\mu_{\theta^{n} \omega}\left(\mathcal{A}_{i t}^{\omega, n} f_{\omega}\right)\right] .
$$

Since $\left\|\mathcal{A}_{i t}^{\omega, n}\right\|_{\infty} \leq\left\|\mathcal{A}_{0}^{\omega, n} \mathbf{1}\right\|_{\infty}=1$ and $\left\|f_{\omega}\right\|_{\infty}$ is integrable it follows that the series $L_{\rho}^{\omega}(\cdot)$ converges uniformly on $\mathbb{R}$. Consider first the non-lattice case. Then by the inversion formula of the Fourier transform (applied with the function $g$ ) and the Fubini theorem, for any $n \geq 1$,

$$
\int \mu_{\theta^{n} \omega}\left(f_{\omega}\left(\xi_{n}^{\omega}\right) g\left(S_{n}^{\theta^{n} \omega}\right)\right) d P(\omega)=\frac{1}{2 \pi} \int_{-b}^{b} \hat{g}(t) \mathbb{E}_{P}\left[\mu_{\theta^{n} \omega}\left(\mathcal{A}_{i t}^{\omega, n} f_{\omega}\right)\right] d t
$$

and therefore,

$$
U_{\rho}(g)=\frac{1}{2 \pi} \int_{-b}^{b} \hat{g}(t) L_{\rho}(t) d t
$$

Next, by (3.3) there exists a constant $C>0$ so that for any $t \in\left[-\delta_{0}, \delta_{0}\right]$,

$$
\sum_{n \geq 1} \mathbb{E}_{P}\left\|\mathcal{A}_{i t}^{\omega, n} f_{\omega}-\lambda_{\omega, n}(i t) \nu_{\omega}(i t)\left(f_{\omega}\right) h_{\theta^{n} \omega}(i t)\right\|_{\alpha, \xi} \leq C
$$

where we used that $\left\|f_{\omega}\right\|_{\alpha, \xi}$ is $P$-integrable and that $\left|\lambda_{\omega, n}(i t)\right|$ is bounded in $\omega, n$ and $t \in\left[-\delta_{0}, \delta_{0}\right]$ (see (3.11)). In fact, (3.3) also implies that the series on the left hand side of (4.19) converges uniformly over $t \in\left[-\delta_{0}, \delta_{0}\right]$. As a consequence, we can write

$$
2 \pi\left(U_{\rho}(g)-V_{\rho}(g)\right)=\int_{\delta_{0}<|t| \leq b} \hat{g}(t) L_{\rho}(t) d t+\int_{-\delta_{0}}^{\delta_{0}} \hat{g}(t) R_{2, \rho}(t) d t
$$


where

$$
R_{2, \rho}(t)=\sum_{n \geq 1} \rho^{n-1} \mathbb{E}_{P} \mu_{\theta^{n} \omega}\left(\mathcal{A}_{i t}^{\omega, n} f_{\omega}-\lambda_{\omega, n}(i t) \nu_{\omega}(i t)\left(f_{\omega}\right) h_{\theta^{n} \omega}(i t)\right)
$$

which converges as $\rho \rightarrow 1$ to $R_{2,1}(t)$ uniformly over $t \in\left[-\delta_{0}, \delta_{0}\right]$. It is clear by (4.19) that $\sup _{t \in\left[-\delta_{0}, \delta_{0}\right]}\left|R_{2,1}(t)\right| \leq C$. We claim next that $L_{\rho}(t)$ converges uniformly on $[-b, b] \backslash\left[-\delta_{0}, \delta_{0}\right]$ towards a limit $R_{1}(t)$ which is a bounded function of $t$ on this domain. This will complete the proof of the lemma in the non-lattice case. Indeed, set $J=[-b, b] \backslash\left[-\delta_{0}, \delta_{0}\right]$ and let $\Delta_{n}=\Delta_{n}(J)$ be the sets from Lemma 4.3. Then by (4.9) and (4.10) there exist constants $d=d(J)>0$ and $u=u(J)>0$ so that $P\left(\Delta_{n}\right) \geq 1-d n^{-\beta}$ and for any $\omega \in \Delta_{n}$,

$$
\sup _{t \in J}\left\|\mathcal{A}_{i t}^{\omega, n}\right\|_{\alpha, \xi} \leq 4 B_{0} \cdot 2^{-u n} .
$$

Therefore, with $\|\cdot\|=\|\cdot\|_{\alpha, \xi}$, for any $t \in J$ and $m \geq 1$ we have

$$
\begin{array}{r}
\sum_{n \geq m}\left|\mathbb{E}_{P} \mu_{\theta^{n} \omega}\left(\mathcal{A}_{i t}^{\omega, n} f_{\omega}\right)\right| \leq \sum_{n \geq m} \mathbb{E}_{P}\left\|f_{\omega}\right\| \cdot\left\|\mathcal{A}_{i t}^{\omega, n}\right\| \\
\leq \sum_{n \geq m} \int_{\Delta_{n}^{c}}\left\|f_{\omega}\right\| \cdot\left\|\mathcal{A}_{i t}^{\omega, n}\right\| d P(\omega)+4 B_{0} \sum_{n \geq m} 2^{-u n} \int_{\Delta_{n}}\left\|f_{\omega}\right\| d P(\omega) \\
\leq B_{J} \sum_{n \geq m} \int_{\Delta_{n}^{c}}\left\|f_{\omega}\right\| d P(\omega)+4 B_{0} \mathbb{E}_{P}\left\|f_{\omega}\right\| \sum_{n \geq m} 2^{-u n}
\end{array}
$$

where $\Delta_{n}^{c}=\Omega \backslash \Delta_{n}$ and $B_{J}$ is defined after (4.11). Set $\|f\|_{p, \alpha, \xi}^{p}:=\int\left\|f_{\omega}\right\|^{p} d P(\omega)<$ $\infty$. Applying the Hölder inequality yields

$$
\int_{\Gamma_{n}^{c}}\left\|f_{\omega}\right\| d P(\omega) \leq d^{1-\frac{1}{p}}\|f\|_{p, \alpha, \xi} n^{-\beta\left(1-\frac{1}{p}\right)} .
$$

Since $\beta\left(1-\frac{1}{p}\right)>1$ the series defined by the above right hand side (with $n=1,2, \ldots$ ) converges, and hence the (uniform) limit of $L_{\rho}(t)$ as $\rho \rightarrow 1$ exists on $\left[-\delta_{0}, \delta_{0}\right]$.

Next, in the lattice case we proceed in a slightly different way. We first rewrite (4.18) as

$$
U_{\rho}(g)=\frac{1}{2 \pi} \int_{-\frac{\pi}{h}}^{\frac{\pi}{h}} r(t) L_{\rho}(t) d t
$$

where we used that $L_{\rho}$ is $\frac{2 \pi}{h}$ periodic which holds true since

$$
\mu_{\theta^{n} \omega}\left(\mathcal{A}_{i t}^{\omega, n} f_{\omega}\right)=\mathbb{E}\left[f_{\omega}\left(\xi_{n}^{\omega}\right) e^{i t S_{n}^{\theta^{n} \omega}}\right]
$$

which is indeed a $\frac{2 \pi}{h}$-periodic function of $t$ in view of the lattice assumption. The proof proceeds now in a similar way taking compact subintevrals $J$ of $\left(-\frac{2 \pi}{h}, \frac{2 \pi}{h}\right)$ which do not contain the origin.

Now, we prove

4.5. Lemma. If $\delta_{0}$ is sufficiently small then there exists an integrable function $R_{3}$ on $\left[-\delta_{0}, \delta_{0}\right] \backslash\{0\}$ so that with $g_{a}(x)=g(x-a)$, for any $a \in \mathbb{R} \backslash\{0\}$,

$$
\lim _{\rho \rightarrow 1} 2 \pi V_{\rho}\left(g_{a}\right)=\int_{-\delta_{0}}^{\delta_{0}} e^{-i t a} R_{3}(t) d t+\frac{\boldsymbol{\mu}(0)(f)}{\gamma} \kappa_{h}(g)\left(\pi+\int_{-\delta_{0} a}^{\delta_{0} a} \frac{\sin t}{t} d t\right)
$$

where in the lattice case $\kappa_{h}$ is the measure assigning unit mass to each point of the lattice $h \mathbb{Z}$, while in the non-lattice case $\kappa_{0}$ is the Lebesgue measure. 
Proof. First, we have $\hat{g}_{a}(t)=e^{-i t a} \hat{g}(t)$ and $\sum_{k} \hat{g}_{a}\left(t+\frac{2 \pi k}{h}\right)=e^{-i t a} r(t)$. Observe that

and therefore,

$$
\rho \lambda_{\theta^{-1} \omega}(i t) f_{\rho}^{\omega}(t)=f_{\rho}^{\theta^{-1} \omega}(t)-\lambda_{\theta^{-1} \omega}(i t) \mu_{\omega}\left(h_{\omega}(i t)\right)
$$

$$
f_{\rho}^{\omega}(t)=\frac{\lambda_{\theta^{-1} \omega}(i t) \mu_{\omega}\left(h_{\omega}(i t)\right)}{1-\rho \lambda_{\theta^{-1} \omega}(i t)}+\frac{f_{\rho}^{\omega}(t)-f_{\rho}^{\theta^{-1} \omega}(t)}{1-\rho \lambda_{\theta^{-1} \omega}(i t)} .
$$

Since $\lambda_{\omega}^{\prime}(0)=\gamma>0$ we obtain from (3.2) that there exists a constant $a_{0}>0$ so that for any sufficiently small $\delta_{0}, t \in\left[-\delta_{0}, \delta_{0}\right]$ and $\frac{1}{2}<\rho \leq 1$,

$$
\left|1-\rho \lambda_{\theta^{-1} \omega}(i t)\right| \geq a_{0}|t|
$$

and so, the above decomposition of $f_{\rho}^{\omega}(t)$ makes sense when $\rho>\frac{1}{2}$ and $t \neq 0$. Set

$$
\mathcal{D}_{\omega, n}(t):=\lambda_{\omega, n}(i t) \mu_{\theta^{n} \omega}\left(h_{\theta^{n} \omega}(i t)\right)-\lambda_{\theta^{-1} \omega, n}(i t) \mu_{\theta^{n-1} \omega}\left(h_{\theta^{n-1} \omega}(i t)\right) .
$$

Then

$$
f_{\rho}^{\omega}(t)-f_{\rho}^{\theta^{-1} \omega}(t)=\sum_{n \geq 1} \rho^{n-1} \mathcal{D}_{\omega, n}(t)
$$

Write

$$
\begin{array}{r}
\mathcal{D}_{\omega, n}(t)=\lambda_{\omega, n}(i t)\left(\lambda_{\theta^{n-1} \omega}(i t)\right)^{-1} \times \\
\left(\mu_{\theta^{n} \omega}\left(h_{\theta^{n} \omega}(i t)\right) \lambda_{\theta^{n-1} \omega}(i t)-\mu_{\theta^{n-1} \omega}\left(h_{\theta^{n-1} \omega}(i t)\right) \lambda_{\theta^{-1} \omega}(i t)\right) .
\end{array}
$$

As in the proof of Lemma 7.2.1 in [13], differentiating the equality $\nu_{\omega}(z)\left(h_{\omega}(z)\right)$ at $z=0$ and using that $h_{\omega}(0) \equiv \mathbf{1}$ and $\nu_{\omega}(z) \mathbf{1}=1$ yields that $\boldsymbol{\mu}_{\omega}(0)\left(h_{\omega}^{\prime}(0)\right)=$ $\nu_{\omega}(0)\left(h_{\omega}^{\prime}(0)\right)=0$. By (3.2) and since $\lambda_{\omega}^{\prime}(0)=\gamma>0$, when $\delta_{0}$ is sufficiently small then the absolute value of the term inside the brackets above does not exceed $c_{0} t^{2}$ for some constant $c_{0}$ which does not depend on $\omega, n$ and $t$. Indeed, this term has bounded second derivatives, it vanishes at $t=0$ and its first derivative at 0 equals 0 . Now, by (4.21) there exists a constant $c_{1}>0$ so that $\left|\lambda_{\theta^{n-1} \omega}(i t)\right| \geq c_{1}$ for any $n \geq 1$ and $t \in\left[-\delta_{0}, \delta_{0}\right]$. We conclude that there exist constants $c_{2}$ and $c_{2}^{\prime}$ so that for any $t \in\left[-\delta_{0}, \delta_{0}\right], 1 \leq q<\infty$ and $n \geq 1$,

$$
\left\|\mathcal{D}_{\omega, n}(t)\right\|_{q} \leq c_{2}^{\prime} t^{2}\left\|\lambda_{\omega, n}(i t)\right\|_{q} \leq c_{2} t^{2}\left(P\left(\Gamma_{n}^{c}\right)\right)^{\frac{1}{q}}+c_{2} t^{2} e^{-n d_{2} t^{2}}
$$

where $\|X\|_{q}=\|X\|_{L^{q}(\Omega, \mathcal{F}, P)}$ for any random variable $X$, the set $\Gamma_{n}^{c}$ is the compliment of the set satisfying (4.6) and (4.7) and we also used (3.11). In particular, by the Hölder inequality for any $Y \in L^{p}(\Omega, \mathcal{F}, P), 1<p \leq \infty$,

$$
E\left|Y \mathcal{D}_{\omega, n}(t)\right| \leq\|Y\|_{p}\left(c_{2} t^{2}\left(P\left(\Gamma_{n}^{c}\right)\right)^{1-\frac{1}{p}}+c_{2} t^{2} e^{-n d_{2} t^{2}}\right) .
$$

Since $\beta\left(1-\frac{1}{p}\right)>1$ and $t^{2} \sum_{n \geq 1} e^{-n d_{2} t^{2}}$ is bounded in $t \in\left[-\delta_{0}, \delta_{0}\right]$, we obtain from (4.23) that

$$
\mathbb{E}_{P}\left|Y\left(f_{\rho}^{\omega}(t)-f_{\rho}^{\theta^{-1} \omega}(t)\right)\right| \leq C\left(1+\|Y\|_{p}\right)
$$

where $C>0$ is a constant which does not depend on $Y$ and $t$. Next, for any $t \in\left[-\delta_{0}, \delta_{0}\right]$ set $\psi^{\omega}(t)=e(t) \lambda_{\theta-1}(i t) \mu_{\omega}\left(h_{\omega}(i t)\right) \nu_{\omega}(i t)\left(f_{\omega}\right)$ where $e(t)=\hat{g}(t)$ in the non-lattice case, while in the lattice case $e(t)=r(t)$. Then by (3.2) we have $\left|\psi^{\omega}(t)\right| \leq C^{\prime}\left\|f_{\omega}\right\|_{\alpha, \xi}$, where $C^{\prime}>0$ is some constant. Moreover,

$$
\psi^{\omega}(0)=e(0) \nu_{\omega}(0)\left(f_{\omega}\right)=\nu(0)(f) \int g(x) d \kappa_{h}(x)
$$


and $\psi^{\omega}(t)=\psi^{\omega}(0)+t \psi_{1}^{\omega}(t)$ for some bounded function $\psi_{1}^{\omega}:\left[-\delta_{0}, \delta_{0}\right] \rightarrow \mathbb{C}$ so that $\mathbb{E}_{P}\left|\psi^{\omega}(t)\right| \leq C^{\prime \prime} \mathbb{E}_{P}\left\|f_{\omega}\right\|_{\alpha, \xi}$ for any $t \in\left[-\delta_{0}, \delta_{0}\right]$, where $C^{\prime \prime}$ is another constant. We also set $\Delta_{\rho}^{\omega}(t)=f_{\rho}^{\omega}(t)-f_{\rho}^{\theta^{-1} \omega}(t)$ and $\phi^{\omega}(t)=e(t) \nu_{\omega}(i t)\left(f_{\omega}\right)$. Then by (4.24) the random variables $\Delta_{\rho}^{\omega}(t)$ are integrable and since $P$ is $\theta$-invariant we have $\mathbb{E}_{P} \Delta_{\rho}^{\omega}(t)=$ 0 . Now, for any $\rho \in\left(\frac{1}{2}, 1\right)$ write,

$$
2 \pi V_{\rho}\left(g_{a}\right)=\int_{-\delta_{0}}^{\delta_{0}} e^{-i t a}\left(F_{\rho}(t)+G_{\rho}(t)\right) d t+J(a, \rho) \kappa_{h}(g) \nu(0)(f)
$$

where

$$
\begin{array}{r}
J(a, \rho)=\int_{-\delta_{0}}^{\delta_{0}} \frac{e^{-i t a}}{1-\rho(1+i \gamma t)} d t \\
F_{\rho}(t)=\mathbb{E}_{P}\left[\frac{\psi^{\omega}(t)}{1-\rho \lambda_{\theta^{-1} \omega}(i t)}-\frac{\psi^{\omega}(0)}{1-\rho(1+i \gamma t)}\right] \\
\text { and } G_{\rho}(t)=\mathbb{E}_{P}\left[\frac{\phi^{\omega}(t) \Delta_{\rho}^{\omega}(t)}{1-\rho \lambda_{\theta^{-1} \omega}(i t)}\right]
\end{array}
$$

and we used (4.20). As in the proof of Lemma VII.3 in [15] we have

$$
\lim _{\rho \rightarrow 1} J(a, \rho)=\gamma^{-1}\left(\pi+\int_{-a \delta_{0}}^{a \delta_{0}} \frac{\sin t}{t} d t\right) .
$$

In order to compete the proof of the lemma in the case discussed above, it is sufficient to show that $F_{\rho}(t)$ and $G_{\rho}(t)$ are bounded in both $t \in\left[-\delta_{0}, \delta_{0}\right] \backslash\{0\}$ and

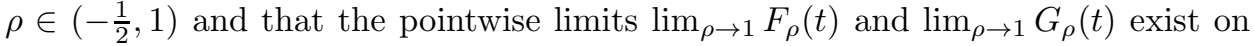
$\left[-\delta_{0}, \delta_{0}\right] \backslash\{0\}$. We first show that the above statement holds true for $F_{\rho}(\cdot)$. First, by (4.21) and since $\left|\psi^{\omega}(t)\right| \leq C^{\prime}\left\|f_{\omega}\right\|_{\alpha, \xi}$, applying the dominated convergence theorem yields that for any $t \in\left[-\delta_{0}, \delta_{0}\right] \backslash\{0\}$,

$$
\lim _{\rho \rightarrow 1} F_{\rho}(t)=\mathbb{E}_{P}\left[\frac{\psi^{\omega}(t)}{1-\lambda_{\theta^{-1}}(i t)}-i \frac{\psi^{\omega}(0)}{\gamma t}\right]
$$

Moreover, for any $t$ and $\rho$ in the above domain,

$$
\begin{array}{r}
\left|F_{\rho}(t)\right|=\left|t \mathbb{E}_{P}\left[\frac{\psi_{1}^{\omega}(t)}{1-\rho \lambda_{\theta^{-1} \omega}(i t)}\right]+\rho \mathbb{E}_{P}\left[\frac{\lambda_{\theta^{-1} \omega}(i t)-1-i \gamma t}{\left(1-\rho \lambda_{\theta^{-1} \omega}(i t)\right)(1-\rho(1+i \gamma t))} \psi^{\omega}(0)\right]\right| \\
\leq C_{0} \mathbb{E}_{P}\left\|f_{\omega}\right\|_{\alpha, \xi}+C_{0}|e(0) \nu(0)(f)| t^{-2} \mathbb{E}_{P}\left|\lambda_{\theta^{-1} \omega}(i t)-1-i \gamma t\right| \\
\leq A_{0}\left(\mathbb{E}_{P}\left\|f_{\omega}\right\|_{\alpha, \xi}+|e(0) \nu(0)(f)|\right)
\end{array}
$$

where we used (3.2) and (4.21) and $C_{0}, A_{0}>0$ are some constants, and we obtain the desired estimate.

Next, in order to show that $G_{\rho}$ converges pointwise on $\left[-\delta_{0}, \delta_{0}\right] \backslash\{0\}$ as $\rho \rightarrow 1$, we will need the following simple result. Let $X_{1}, X_{2}, \ldots$ be a sequence of random variables so that $\sum_{n \geq 1} \mathbb{E}\left|X_{n}\right|<\infty$. Let $Y_{\rho}, \rho \in\left(\frac{1}{2}, 1\right]$ be a collection of random variables so that $\left\|Y_{\rho}-Y_{1}\right\|_{L^{\infty}}$ converges to 0 as $\rho \rightarrow 1$ and $Y_{1}$ is bounded. Then

$$
\lim _{\rho \rightarrow 1} \sum_{n \geq 1} \rho^{n-1} \mathbb{E} X_{n} Y_{\rho}=\sum_{n \geq 1} \mathbb{E} X_{n} Y_{1} .
$$

Using (4.23) with $Y \equiv 1$ and then the above result with $X_{n}=\phi^{\omega}(t) \mathcal{D}_{\omega, n}(t)$ and

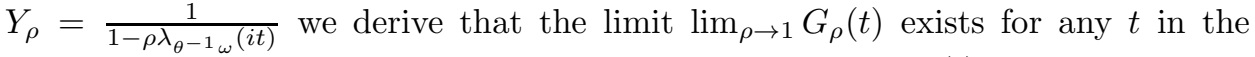
above domain. To complete the proof, we will show that $G_{\rho}(t)$ is bounded when considered as a function of $(\rho, t) \in\left(\frac{1}{2}, 1\right) \times\left[-\delta_{0}, \delta_{0}\right]$. Indeed, let $(\rho, t)$ be in the 
latter domain. In the following arguments all the constants will depend only on $\delta_{0}$ (and not on $\omega, \rho$ and $t$ ). Since $\mathbb{E}_{P} \Delta_{\rho}^{\omega}(t)=0$ and $\phi_{\omega}(0)$ does not depend on $\omega$, for we can write

$$
\begin{array}{r}
G_{\rho}(t)=\mathbb{E}_{P} \frac{\phi^{\omega}(t) \Delta_{\rho}^{\omega}(t)}{1-\rho \lambda_{\theta^{-1} \omega}(i t)} \\
=\mathbb{E}_{P}\left[\phi^{\omega}(t) \Delta_{\rho}^{\omega}(t)\left(\left(1-\rho \lambda_{\theta^{-1} \omega}(i t)\right)^{-1}-(1-\rho(1+i \gamma t))^{-1}\right)\right] \\
+\mathbb{E}_{P}\left[\left(\phi^{\omega}(t) \Delta_{\rho}^{\omega}(t)-\phi^{\omega}(0) \Delta_{\rho}^{\omega}(t)\right)(1-\rho(1+i \gamma t))^{-1}\right]:=I_{1}+I_{2} .
\end{array}
$$

By (4.24), (3.2) and our assumption that $\beta\left(1-\frac{1}{p}\right)>1$ we have

$$
\mathbb{E}_{P}\left|\phi^{\omega}(t) \Delta_{\rho}^{\omega}(t)\right| \leq C_{1} \mathbb{E}_{P}\left[\left\|f_{\omega}\right\|_{\alpha, \xi}\left|\Delta_{\rho}^{\omega}(t)\right|\right] \leq C_{2}
$$

for some constants $C_{1}, C_{2}>0$. Moreover, by (3.2) and (4.21) there exist constants $C_{3}>0$ and $C_{3}^{\prime}>0$ so that

$$
\left|\left(1-\rho \lambda_{\theta^{-1} \omega}(i t)\right)^{-1}-(1-\rho(1+i \gamma t))^{-1}\right| \leq C_{3}^{\prime} t^{-2}\left|\lambda_{\theta^{-1} \omega}(i t)-(1+i \gamma t)\right| \leq C_{3} .
$$

We conclude that $\left|I_{1}\right| \leq C$ for some constant $C$. Next, by (3.2), there exists a constant $C_{4}>0$ so that $\left|\phi^{\omega}(t)-\phi^{\omega}(0)\right| \leq C_{4}\left(1+\left\|f_{\omega}\right\|_{\alpha, \xi}\right)|t|$, and therefore, using also (4.24),

$$
\left|I_{2}\right|=|1-\rho(1+i \gamma t)|^{-1}\left|\mathbb{E}_{P}\left[\left(\phi^{\omega}(t)-\phi^{\omega}(0)\right) \Delta_{\rho}^{\omega}(t)\right]\right| \leq C_{5}
$$

where $C_{5}$ is another constant. The proof of the lemma is complete now in the case when $\mu_{\omega}\left(h_{\omega}^{\prime}(0)\right)$ does not depend on $\omega$.

The completion of the proof of Theorem 2.8 is done similarly to [15] (see references therein). Let $g \in \mathscr{H}_{1}$ be dominated by a positive member of $\mathscr{H}_{1}$. If $g$ is positive then $U(g)=\lim _{\rho \rightarrow 1} U_{\rho}(g)$ which is finite in view of Lemmas 4.4 and 4.5 . Thus $U$ is a Radon measure on $\mathbb{R}$ (see [15] and references therein). Next, for any $a \in \mathbb{R}$ the integral $U\left(g_{a}\right)$ is defined and is the limit of $U_{\rho}\left(g_{a}\right)$ as $\rho \rightarrow 1$. Therefore by Lemmas 4.4 and 4.5 .

$$
U\left(g_{a}\right)-\frac{\nu(0)(f) \kappa_{h}(g)}{\gamma} \frac{1}{2 \pi}\left(\pi+\int_{-a \delta_{0}}^{a \delta_{0}} \frac{\sin t}{t} d t\right)=\widehat{Q_{1}}(a)+\widehat{Q_{2}}(a)+\frac{1}{2 \pi} \widehat{R_{3}}(a)
$$

where $Q_{i}(t)=R_{i}(t) e(t), i=1,2$ and we set $R_{1}(t)=R_{3}(t)=0$ for any $t \in\left(-\delta_{0}, \delta_{0}\right)$ and $R_{2}(t)=0$ for any $t \in \mathbb{R} \backslash\left[-\delta_{0}, \delta_{0}\right]$. The functions $Q_{1}, Q_{2}$ and $R_{3}$ are integrable, and so, by the Riemann-Lebesgue Lemma, the right hand side of (4.25) converges to 0 as $|a| \rightarrow \infty$. Finally, as in [15,

$$
\left|\mathbb{I}(a \geq 0)-\frac{1}{2 \pi}\left(\pi+\int_{-a \delta_{0}}^{a \delta_{0}} \frac{\sin t}{t} d t\right)\right| \leq 2|a|^{-1}
$$

for any nonzero $a \in \mathbb{R}$, and the proof of Theorem 2.8 is complete.

\section{MiXing CONDitions}

In this section we will show that conditions (2.7) and (2.8) in Assumption 2.6 hold true for shift spaces generated by certain mixing processes and for (natural invertible extensions of) certain dynamical systems. We will focus only the invertible case, and the conditions will hold true in the non-invertible case by considering functions of the 0-the coordinate (in the natural extension) and using Section 4.1. We will always assume that Assumption 2.3 holds true. We begin with 
5.1. Proposition. Suppose that there exists a measure $\mu$ on $\mathcal{X}$ and functions $r_{\omega}: \mathcal{X} \rightarrow \mathbb{R}$ so that $\boldsymbol{\mu}_{\omega}(0)=r_{\omega} \mu$ and all $r_{\omega}$ 's take values at some finite interval $(m, M) \subset(0, \infty)$. Moreover, assume that there exist constants $d_{1}>0$ and $\beta>0$ so that for any sufficiently large $k$ and $n \geq 1$,

$$
P\left\{\omega: \sum_{j=0}^{n-1} \tilde{V}_{k}\left(\theta^{j k} \omega\right) \leq d_{1} k n\right\} \leq \frac{d_{2}(k)}{n^{\beta}}
$$

where $\tilde{V}_{k}(\omega)=\mathbb{E}_{\mu}\left(S_{k}^{\omega} \bar{u}\right)^{2}$ and $d_{2}(k)$ is a constant which depends only on $k$. Then (2.7) holds true. In particular, (2.7) holds true when polynomial concentration inequalities of the form

$$
P\left\{\omega:\left|\sum_{n=0}^{n-1} \tilde{V}_{k}\left(\theta^{j k} \omega\right)-n \mathbb{E}_{P} \tilde{V}_{k}\right| \geq \varepsilon n\right\} \leq \frac{d(k, \varepsilon)}{n^{\beta}}
$$

hold true, where $\varepsilon>0$ and $d(k, \varepsilon)$ is a constant which depends only $k$ and $\varepsilon$.

Proof. Let $1 \leq k \leq n$, set

$$
S(\omega, j)=S_{j k}^{\theta^{j k} \omega} \bar{u}, j=0,1, \ldots,[n / k]-1
$$

and $S(\omega,[n / k])=S_{n-k[n / k]}^{\theta^{[n / k]}} \bar{u}$, where $S_{0}^{\omega}$ is defined to be 0 . Since $\left\|u_{\omega}\right\|_{\alpha, \xi}$ is a bounded random variable and $\nu(0)=\boldsymbol{\mu}(0)$ is $T$-preserving, applying Lemma 5.10.4 in [13] together with (3.3), namely, the uniform in $\omega$ exponential decay of correlations, yields that

$$
\begin{aligned}
V_{n}(\omega) & =\mathbb{E}_{\nu_{\omega}(0)}\left(\sum_{j=0}^{[n / k]} S(\omega, j)\right)^{2}=\sum_{0 \leq j_{1}, j_{2} \leq[n / k]} \operatorname{cov}_{\nu_{\omega}(0)}\left(S\left(\omega, j_{1}\right), S\left(\omega, j_{2}\right)\right) \\
& \geq \sum_{j=0}^{\left[\frac{n}{k}\right]-1} \mathbb{E}_{\nu_{\omega}(0)}(S(\omega, j))^{2}-A\left(\frac{n}{k}+1\right)=\sum_{j=0}^{\left[\frac{n}{k}\right]-1} V_{k}\left(\theta^{j k} \omega\right)-A\left(\frac{n}{k}+1\right)
\end{aligned}
$$

where $A>0$ is some positive constant. It follows from the assumption about the densities $r_{\omega}$ that

$$
V_{n}(\omega) \geq \frac{m}{M} \sum_{j=0}^{\left[\frac{n}{k}\right]-1} \tilde{V}_{k}\left(\theta^{j k} \omega\right)-A\left(\frac{n}{k}+1\right) .
$$

Taking a sufficiently large $k$ we derive that on the complement of the set whose probability is estimated in (5.1) we have

$$
V_{n}(\omega) \geq \frac{M d_{1} n}{m}-A\left(\frac{n}{k}+1\right) .
$$

If $k$ is sufficiently large then the above right hand side is not less than $c n$ for some constant $c>0$, and so condition (2.7) is satisfied. Finally, (5.2) implies (5.1) since $\mathbb{E}_{P} \tilde{V}_{k} \geq \frac{m}{M} \mathbb{E}_{P} V_{k}$ and $\lim _{k \rightarrow \infty} k^{-1} \mathbb{E}_{P} V_{k}=\sigma^{2}>0$

Note that when $\boldsymbol{\mu}_{\omega}=\nu_{\omega}(0)$ does not depend on $\omega$ then the first assumption in Proposition is satisfied with $\mu=\nu_{\omega}(0)$ and $r_{\omega}=1$. The measures $\boldsymbol{\mu}_{\omega}$ do not depend on $\omega$ when only the function $u_{\omega}$ is random. These measures do no depend on $\omega$ also in the non-invertible case considered in Section 2.2, since, in the extension, we have $\nu_{\omega}(0)=\boldsymbol{\mu}_{\omega}(0)=\kappa$. When $\boldsymbol{\mu}_{\omega}$ depends on $\omega$, then, in the circumstances of Proposition 3.2 the first assumption is satisfied with $r_{\omega}=\mathbf{h}_{\omega}(0)$ and $\mu=\mathbf{m}$, since 
the function function $\mathbf{h}_{\omega}(0)$ is bounded from above and below by positive constants not depending on $\omega$ (see Section 5.12 in [13]).

Henceforth, we assume that all the conditions of Proposition 5.1 are satisfied. We provide now sufficient conditions for (5.2) and (2.8) to hold true in two situations. First consider the case when $(\Omega, \mathcal{F}, P, \theta)=\left(\hat{\Omega}_{0}, \hat{\mathcal{F}}_{0}, \hat{P}_{0}, \hat{\vartheta}\right)$ is the natural invertible extension (described in 4.1) of a measure preserving system $\left(\Omega_{0}, \mathcal{F}_{0}, P_{0}, \vartheta\right)$. Note that $\theta$ has a periodic point if and only if $\vartheta$ has a periodic point. We assume here that $\Omega_{0}$ is equipped with a metric $d_{0}$ so that $\operatorname{diam} \Omega_{0} \leq 1$ and $\mathcal{F}_{0}$ contains the appropriate Borel $\sigma$-algebra. Let the metric $d$ on $\Omega_{0}^{\mathbb{Z}}$ be defined by

$$
d(a, b)=\sum_{n \in \mathbb{Z}} 2^{-|n|} d_{0}\left(a_{n}, b_{n}\right) \text { for any } a=\left(a_{n}\right)_{n \in \mathbb{Z}} \text { and } b=\left(b_{n}\right)_{n \in \mathbb{Z}} .
$$

Let $\iota: \Omega \rightarrow \Omega_{0}^{\mathbb{Z}}$ be the inclusion map given by $\iota \omega=\omega$. We also need the following

5.2. Assumption. The function $u_{\omega}$ and the transformation $T_{\omega}$ have the form $u_{\omega}=\boldsymbol{u}_{\iota \omega}$ and $T_{\omega}=\boldsymbol{T}_{\iota \omega}$, where $\boldsymbol{u}_{v}$ and $\boldsymbol{T}_{v}$ are Hölder continuous functions of the variable $v \in\left(\Omega_{0}^{\mathbb{Z}}, d\right)$ with respect to the norm $\|\cdot\|_{\alpha, \xi}$ and the metric $d\left(\boldsymbol{T}_{v_{1}}, \boldsymbol{T}_{v_{2}}\right)=\sup _{x \in \mathcal{X}} \rho\left(\boldsymbol{T}_{v_{1}} x, \boldsymbol{T}_{v_{2}} x\right)$, respectively.

This assumption includes the case when $u_{\omega}$ and $T_{\omega}$ depend only on the first coordinate $\zeta_{0}$ and are Hölder continuous functions of this coordinate when considered as functions on $\left(\Omega_{0}, d_{0}\right)$ (as in Section 4.1). Under Assumption 5.2, the functions $\tilde{V}_{k}, k \geq 1$ have the form $\tilde{V}_{k}(\omega)=\tilde{\boldsymbol{V}}_{k}(\boldsymbol{\iota} \omega)$, where $\tilde{\boldsymbol{V}}_{k}$ is a Hölder continuous function on $\left(\Omega_{0}^{\mathbb{Z}}, d\right)$. Therefore, for any $N>0$ there exists a Hölder continuous function $\tilde{\boldsymbol{V}}_{k, N}$, which depends only on the coordinates whose indexes lie in $\{-N, \ldots, 0, \ldots N\}$, so that

$$
\sup _{v}\left|\tilde{\boldsymbol{V}}_{k}(v)-\tilde{\boldsymbol{V}}_{k, N}(v)\right| \leq C_{k} 2^{-N}
$$

where $C_{k}$ is some constant which depends only on $k$.

Relying on (5.3) and the results in [5] (see also [6]) and [14, in the above circumstances we have

5.3. Proposition. Let $\beta>0$. Then conditions (5.1) and (2.8) hold true when $\left(\Omega_{0}, \mathcal{F}_{0}, P_{0}, \vartheta\right)$ is either a topologically mixing subshift of finte type, a Young tower with at least one periodic point, with tails of order $n^{-\beta-1}$ or when $\vartheta^{n}(\omega)=\xi_{n}(\omega)$, where $\xi_{0}, \xi_{1}, \xi_{2}, \ldots$ is the stationary vector valued processes satisfying the mixing and approximation conditions from [14, assuming that $\vartheta$ has at least one periodic point, $\Omega_{0}$ is a metric space and $P_{0}(A)>0$ for all open sets $A$.

This proposition holds true since (by either [5] or [14]) all the maps mentioned there satisfy (5.2) and that for any Lipschitz function $f: \Omega_{0}^{\mathbb{Z}} \rightarrow \mathbb{R}$ there exist constants $d(\varepsilon), \varepsilon>0$ so that for any $n \geq 1$,

$$
P\left\{\omega:\left|\sum_{n=0}^{n-1} f\left(\theta^{j} \omega\right)-n \mathbb{E}_{P} f\right| \geq \varepsilon n\right\} \leq \frac{d(\varepsilon)}{n^{\beta}} .
$$

This inequality implies (2.8) since the indicator function of any ball $B(v, r) \subset \Omega_{0}^{\mathbb{Z}}$ can be approximated from below by a Lipschitz function $f$ which takes the constant value 1 on $B\left(v, \frac{1}{2} r\right)$ (see, for instance, Section 1.2.9 in [13]).

Note that when $\vartheta$ is a Young tower the requirement of having a peridoic point is just the assumption that the function $\vartheta^{R} \mid \Gamma: \Gamma \rightarrow \Gamma$ has at least one periodic 
point, which is not too restrictive. Here $\Gamma$ is the (hyperbolic) base set and $R$ is the corresponding return time function.

Next, let $\zeta=\left\{\zeta_{n}, n \geq 0\right\}$ be a stationary sequence of random variables defined on a probability space $\left(\Omega_{0}, \mathcal{F}_{0}, P_{0}\right)$ taking values on some metric space $\left(\mathcal{Y}, d_{0}\right)$ so that $\operatorname{diam} \mathcal{Y} \leq 1$. Let $(\Omega, \mathcal{F}, P, \theta)$ be the natural associated shift, namely, $\Omega=\mathcal{Y}^{\mathbb{Z}}$, $\mathcal{F}$ is the product $\sigma$-algebra, $P$ is given by

$$
P\left\{\left(y_{j}\right)_{j \in \mathbb{Z}}: y_{i} \in A_{i} ;|i| \leq N\right\}=P_{0}\left(\zeta_{i} \in A_{i-N} ; 0 \leq i \leq 2 N\right)
$$

and $\theta$ is the two sided shift. Let $d$ be the metric on $\Omega$ given by $d\left(\left\{a_{n}\right\},\left\{b_{n}\right\}\right)=$ $\sum_{n \in \mathbb{Z}} 2^{-|n|} d_{0}\left(a_{n}, b_{n}\right)$. We assume here that $\zeta$ is stretched exponentially $\alpha$-mixing, namely that there exist constants $a, b, c>0$ so that for any $n \geq 1, k \geq 1, A \in$ $\sigma\left\{\zeta_{1}, \ldots, \zeta_{k}\right\}$ and $B \in \sigma\left\{\zeta_{i}: i \geq k+n\right\}$,

$$
\left|P_{0}(A \cap B)-P_{0}(A) P_{0}(B)\right| \leq a e^{-b n^{c}} .
$$

We first have

5.4. Proposition. (i) Suppose that the functions $\omega \rightarrow u_{\omega}$ and $\omega \rightarrow T_{\omega}$ are Hölder continuous with respect to the norm $\|\cdot\|_{\alpha, \xi}$ and the metric $d\left(T_{\omega}, T_{\omega^{\prime}}\right)=$ $\sup _{x \in \mathcal{X}} \rho\left(T_{\omega} x, T_{\omega^{\prime}}(x)\right)$, respectively. Then the functions $\tilde{V}_{k}$ are Hölder continuous and condition (5.2) holds true with any $\beta>0$.

(ii) Let $\mathcal{J} \subset \mathbb{Z}$ be a finite set and let $\pi_{\mathcal{J}}: \Omega \rightarrow \mathcal{Y}^{J}$ be the projection corresponding to the coordinates indexed by members of $\mathcal{J}$. Then condition (5.2) also holds true (with any $\beta>0$ ) when $u_{\omega}=u_{\pi_{\mathcal{J}} \omega}$ and $T_{\omega}=T_{\pi_{\mathcal{J}} \omega}$ depend only on the coordinates in places indexed by the members of $J$, without assuming that $\tilde{V}_{k}$ 's are continuous.

Proposition 5.4 follows from the so-called method of cumulants, see, for instance, [27, [19, 9], 8] or [14] (in the case $\ell=1$ ). The mixing condition (5.4) holds true, for instance, when $\zeta_{n}=\zeta_{0} \circ \vartheta^{n}$ and $\zeta_{0}$ is measurable with respect to a Markov partition corresponding to either a Young tower with stretched exponentially tails (see [16] or 17] for verification of (5.4) ) or a topologically mixing subshift of finite type (see [3]), or has the form $\zeta_{n}=f\left(\Upsilon_{n}\right)$ when $\Upsilon_{n}, n \geq 1$ is a geometrically ergodic Markov chain or a Markov chain satisfying the Doeblin condition (see [2]). Note that the cases discussed in Proposition 5.4 include the case when $T_{\omega}^{n}=T_{\zeta_{n}} \circ T_{\zeta_{n-1}} \circ \cdots \circ T_{\zeta_{1}}$, namely the case when the compositions of the maps $T_{\omega}$ are taken along stationary and sufficiently fast mixing process.

In the above circumstances existence of a periodic points is trivial (see the last paragraph of Section 11), and the question is whether (2.8) holds true.

5.5. Proposition. Condition (2.8) holds true with $\omega_{0}=(\ldots a, a, a, \ldots), a=\left(a_{i}\right) \in$ $\mathcal{Y}^{n_{0}}$ when

$$
P_{0}\left(\zeta_{i+(j-1) n_{0}} \in A_{i, j} ; i=0,1, \ldots, n_{0}-1,1 \leq j \leq s\right)>0
$$

for any open sets $A_{i, j}$ so that $a_{i} \in A_{i, j}$ for all $i$ and $j$.

Proposition 5.5 holds true since in its circumstances (by the method of cumulants), for any open set $B$ which depends only finite number of coordinates and $\beta>0$, there exist positive constants $c_{\beta}(\varepsilon), \varepsilon>0$ so that for any $n \geq 1$,

$$
P\left\{\omega:\left|\sum_{j=0}^{n-1} \mathbb{I}_{B}\left(\theta^{j} \omega\right)-n P(B)\right| \geq \varepsilon n\right\} \leq \frac{c_{\beta}(\varepsilon)}{n^{\beta}} .
$$


In particular, we can consider Markov chains with finite number of states and more generality Markov chains with positive densities $p(x, y)$ around $\left(a_{i}, a_{i+1}\right), i=$ $1, \ldots, n_{0}-1$, whose stationary measure assigns positive mass to open sets. The proposition also holds true when $\zeta_{0}$ is measurable with respect to an appropriate Markov partition since then the non-empty intersection $\cap_{0 \leq i<n_{0}} \cap_{1 \leq j \leq s}$ $\vartheta^{-(j-1) n_{0}+i} A_{i, j}$ has positive $P_{0}$-measure.

\section{Additional Results}

6.1. Non-continuous functions. We explain here how to obtain all the results stated in Section 2 when $\phi_{\omega}$ and $u_{\omega}$ are Hölder continuous only on some pieces of $\mathcal{X}$. First, under Assumption 2.2, $P$-a.s. for any $n \geq 1$ and $x, x^{\prime} \in \mathcal{X}$ with $\rho\left(x, x^{\prime}\right)<\xi$ we can write

$$
\left(T_{\omega}^{n}\right)^{-1}\{x\}=\left\{y_{1}, \ldots, y_{k}\right\} \text { and }\left(T_{\omega}^{n}\right)^{-1}\left\{x^{\prime}\right\}=\left\{y_{1}^{\prime}, \ldots, y_{k}^{\prime}\right\}
$$

where

$$
k=k_{\omega, x, n}=\left|\left(T_{\omega}^{n}\right)^{-1}\{x\}\right| \leq D_{\omega, n}:=\prod_{i=0}^{n-1} D_{\theta^{i} \omega},
$$

$|\Gamma|$ denotes the cardinality of a finite set $\Gamma$ and with $\gamma_{\omega, i}=\prod_{s=0}^{i-1} \gamma_{\theta^{s} \omega}$,

$$
\rho\left(T_{\omega}^{j} y_{i}, T_{\omega}^{j} y_{i}^{\prime}\right) \leq\left(\gamma_{\theta^{j} \omega, n-j}\right)^{-1} \rho\left(x, x^{\prime}\right)
$$

for any $1 \leq i \leq k$ and $0 \leq j<n$.

Let $H_{\omega}$ be a random variable and let $\psi_{\omega} \in \mathcal{H}^{\alpha, \xi}$ be so that $v_{\alpha, \xi}\left(\psi_{\omega}\right) \leq H_{\omega}$. Then by Lemma 5.1.4 in [13], for any $n \geq 1, x, x^{\prime} \in \mathcal{X}$ with $\rho\left(x, x^{\prime}\right)<\xi$ and $1 \leq i \leq k$,

$$
\left|S_{n}^{\omega} \psi\left(y_{i}\right)-S_{n}^{\omega} \psi\left(y_{i}^{\prime}\right)\right| \leq \rho^{\alpha}\left(x, x^{\prime}\right) \sum_{j=0}^{n-1} H_{\theta^{j} \omega}\left(\gamma_{\theta^{j} \omega, n-j}\right)^{-\alpha}
$$

where $y_{1}, \ldots, y_{k}$ and $y_{1}^{\prime}, \ldots, y_{k}^{\prime}$ satisfy (6.1) and (6.2). Not only members of $\mathcal{H}_{\alpha, \xi}$ satisfy (6.3). For instance, when $\mathcal{X}$ is is a $C^{2}$-compact Riemanian manifold and there exist a finite collection of disjoint rectangles $\left\{I_{j}\right\}$ so that $T_{\omega} \mid I_{j}: I_{j} \rightarrow \mathbb{S}^{1}$ is an expanding diffeomorphism for each $j$, then (6.3) also holds true with some $H_{\omega}$ for functions $\psi_{\omega}$ which are only Hölder continuous when restricted to each one of the $I_{j}$ 's. Perhaps the most interesting case is when $\psi_{\omega}=-\ln \left(\frac{d\left(T_{\omega}\right)_{*} \mathbf{m}}{d \mathbf{m}}\right)$ where $\mathbf{m}$ is the normalized volume measure. This includes, of course, the case when $\mathcal{X}=\mathbb{S}^{1}$, $\mathbf{m}$ is the Lebesgue measure and $I_{j}$ 's are disjoint arcs (namely, the classical case of random distance expanding maps on the unit interval).

We have the following

6.1. Theorem. Suppose that all the conditions of Theorems 2.4. 2.7 and 2.8 hold true, except for the ones concerning $\left\|\phi_{\omega}\right\|_{\alpha, \xi}$ and $\left\|u_{\omega}\right\|_{\alpha, \xi}$. Assume that $\phi_{\omega}$ and $u_{\omega}$ satisfy (6.3) with some bounded random variable $H_{\omega}$. Then all the results stated in Theorems 2.4. 2.7 and 2.8 hold true.

This theorem is proved exactly as in Section 4 since all the results from [13] that we applied hold true when (6.3) holds with bounded $H_{\omega}$ 's. In this case, the continuity condition in Assumption 2.5 will be satisfied when the maps $\omega \rightarrow \phi_{\omega}, u_{\omega}$ are continuous with respect to the supremum norm and the differences $S_{n} \phi_{\omega_{1}}-S_{n} \phi_{\omega_{2}}$ and $S_{n} u_{\omega_{1}}-S_{n} u_{\omega_{2}}$ satisfy (6.3) with some constant $H=H\left(\omega_{1}, \omega_{2}\right)$ so that $\lim _{\omega_{1}, \omega_{2} \rightarrow \theta^{k} \omega_{0}} H\left(\omega_{1}, \omega_{2}\right)=0, k=0,1, \ldots, n_{0}-1$. When $T_{\omega}$ is locally constant 
around the points in the orbit of the periodic point $\omega_{0}$ then, in the examples discussed after (6.3), this condition means that restrictions of $\phi_{\omega}$ and $u_{\omega}$ to each one of the $I_{j}$ 's is a continuous function of $\omega$ at $\omega=\theta^{j} \omega_{0}, 0 \leq j<n_{0}$ with respect to the Hölder norm $\|\cdot\|_{\alpha, \xi}$.

6.2. Non-identical fibers. Let $\mathcal{E} \subset \mathcal{F} \times \mathcal{B}$ be a measurable set such that the fibers $\mathcal{E}_{\omega}=\{x \in \mathcal{X}:(\omega, x) \in \mathcal{E}\}, \omega \in \Omega$ are compact. The latter yields (see [7] Chapter III) that the mapping $\omega \rightarrow \mathcal{E}_{\omega}$ is measurable with respect to the Borel $\sigma$-algebra induced by the Hausdorff topology on the space $\mathcal{K}(\mathcal{X})$ of compact subspaces of $\mathcal{X}$ and the distance function $d\left(x, \mathcal{E}_{\omega}\right)$ is measurable in $\omega$ for each $x \in \mathcal{X}$. Furthermore, the projection map $\pi_{\Omega}(\omega, x)=\omega$ is measurable and it maps any $\mathcal{F} \times \mathcal{B}$-measurable set to a $\mathcal{F}$-measurable set (see "measurable projection" Theorem III.23 in [7]). Denote by $\mathcal{P}$ the restriction of $\mathcal{F} \times \mathcal{B}$ on $\mathcal{E}$. Let

$$
\left\{T_{\omega}: \mathcal{E}_{\omega} \rightarrow \mathcal{E}_{\theta \omega}, \omega \in \Omega\right\}
$$

be a collection of continuous bijective maps between the metric spaces $\mathcal{E}_{\omega}$ and $\mathcal{E}_{\theta \omega}$ so that the map $(\omega, x) \rightarrow T_{\omega} x$ is measurable with respect to $\mathcal{P}$ and each $T_{\omega}$ is topologically exact and has the pairing property (namely, appropriate versions of Assumptions 2.3 and 2.2 hold true, see Chapter 5 of [13] for the precise formulations).

According to Lemma 4.11 in [24] (applied with $r=\xi$ ), there exists an integer valued random variable $L_{\omega} \geq 1$ and $\mathcal{F}$-measurable functions $\omega \rightarrow x_{\omega, i} \in \mathcal{X}, i=$ $1,2,3, \ldots$ so that $x_{\omega, i} \in \mathcal{E}_{\omega}$ for each $i$ and

$$
\bigcup_{k=1}^{L_{\omega}} B_{\omega}\left(x_{\omega, k}, \xi\right)=\mathcal{E}_{\omega}, \quad P \text {-a.s. }
$$

Suppose that $L_{\omega}$ is bounded. Then the proof of Theorem 2.4 proceeds exactly as in Section 4 since Theorem 3.1 and all the other results stated in Section 3 hold true.

The role of the condition that $\mathcal{E}_{\omega}$ does not depend on $\omega$ in the proofs of Theorems 2.7 and 2.8 is only to insure that the operators $\mathcal{L}_{i t}^{\omega}$ are defined on the same space when $\omega$ lies in some neighborhood of one of the members of the (periodic) orbit of $\omega_{0}$. The proofs of Theorems 2.7 and 2.8 proceed similarly when there exist open neighborhoods $U_{j}$ of $\omega_{j}:=\theta^{j} \omega_{0}, j=0,1, \ldots, n_{0}-1$ so that $\mathcal{E}_{\omega}=\mathcal{E}_{\omega_{j}}$ for any $\omega \in U_{j}$, namely, when $\mathcal{E}$ is a product set only in neighborhoods of points belonging to the periodic orbit of $\omega_{0}$. In fact, the proof is carried out similarly when for any $j$ and $\omega \in U_{j}$ there exists a bilipschitz homomorphism $\varphi_{\omega, \omega_{j}}: \mathcal{E}_{\omega_{j}} \rightarrow \mathcal{E}_{\omega}$, whose Lipschitz constant is bounded in $\omega$, and for any compact set $J$ and $j=0,1, \ldots, n_{0}-1$,

$$
\lim _{\omega \rightarrow \omega_{j}} \sup _{t \in J} \sup _{g \in \mathcal{H}_{\omega}^{\alpha, \xi}:\|g\|_{\alpha, \xi}=1}\left\|\mathcal{L}_{i t}^{\omega} g_{\omega}-\left(\mathcal{L}_{i t}^{\omega_{j}}\left(g \circ \varphi_{\omega, \omega_{j}}\right)\right) \circ \varphi_{\omega_{j+1}, \theta \omega}\right\|_{\alpha, \xi}=0 .
$$

6.3. Markov chains with transfer (transition) operators. Suppose that $(\Omega, \mathcal{F}, P, \theta)$ is invertible. Let $\mu_{\omega}$ be a (measurable in $\omega$ ) probability measure on $\mathcal{E}_{\omega}$ and let $\xi_{n}^{\theta^{-n} \omega}, n \geq 0$ be a Markov chain with initial distribution $\mu_{\omega}$ whose $n$-th step operator is given by $\mathcal{A}_{0}^{\theta^{-n}} \omega, n$. Set

$$
S_{n}^{\omega}=\sum_{j=0}^{n-1} u_{\theta^{-j} \omega}\left(\xi_{j}^{\theta^{-j} \omega}\right) .
$$


Let $S_{n}$ be the random variable generated by drawing $\omega$ according to $P$ and taking on the fibers the distribution of $S_{n}^{\omega}$, namely the random variable whose characteristic function is given by

$$
\mathbb{E} e^{i t S_{n}}=\int \mu_{\omega}\left(\mathcal{A}_{i t}^{\theta^{-n} \omega, n} \mathbf{1}\right) d P(\omega)
$$

Then the appropriate versions of Theorems 2.4 and 2.7 are proved for the sequence of random variables $S_{n}, n \geq 1$ exactly as in Section 4 . As for the renewal theorem, the arguments in Section 4.7 yield the following

6.2. Theorem. Suppose that Assumptions 2.3, 2.5 and 2.6 hold true, where in the last assumption we require that $\beta>1$. Moreover, assume that $\boldsymbol{\mu}_{\omega}(0)\left(u_{\omega}\right)=\gamma>0$ does not depend on $\omega$. Let $f_{\omega} \in \mathcal{H}_{\alpha, \xi}^{\omega}$ be a positive function so that $\boldsymbol{\mu}_{\omega}(0)\left(f_{\omega}\right)=$ $\boldsymbol{\mu}(0)(f)$ does not depend on $\omega$ and that $\left\|f_{\omega}\right\|_{\alpha, \xi} \in L^{p}(\Omega, \mathcal{F}, P)$ for some $1<p \leq \infty$ so that $\beta\left(1-\frac{1}{p}\right)>1$. For any Borel measurable set $B \subset \mathbb{R}$ set

$$
\begin{gathered}
U(B)=U_{\mu, f}(B)=\sum_{n \geq 1} \mathbb{E}\left[f\left(S_{n}-S_{n-1}\right) \mathbb{I}_{B}\left(S_{n}\right)\right] \\
\quad=\sum_{n \geq 1} \int \mu_{\theta^{-n} \omega}\left(f_{\theta^{-n} \omega}\left(\xi_{n}^{\theta^{-n} \omega}\right) \mathbb{I}_{B}\left(S_{n}^{\omega}\right)\right) d P(\omega)
\end{gathered}
$$

where $\mathbb{I}_{B}$ is the indicator function of the set $B$. Then in both lattice and non-lattice cases $U$ is a Radon measure on $\mathbb{R}$ so that $\int|g| d U<\infty$ for any $C_{4 \downarrow}(\mathbb{R})$. Moreover if either $\mu_{\omega}\left(h_{\omega}^{\prime}(0)\right)$ or $\nu_{\omega}^{\prime}(0)\left(f_{\omega}\right)$ do not depend on $\omega$ then (2.9) holds true for any function $g \in C_{4 \downarrow}(\mathbb{R})$.

In the proof of Lemma 7.2.1 in [13] we showed that $\nu_{\omega}(0)\left(h_{\omega}^{\prime}(0)\right)=0$ and so we can always take $\mu_{\omega}=\nu_{\omega}(0)=\boldsymbol{\mu}_{\omega}(0)$. Since $\nu_{\omega}(z) \mathbf{1}=0$ we can always take $f_{\omega} \equiv \mathbf{1}$ and then choose any $\mu_{\omega}$ for this $f$. In the case when $\nu_{\omega}^{\prime}(0)\left(f_{\omega}\right)$ does not depend on $\omega$ the arguments in Section 4.7 are modified as follows. We first write

$$
\mathbb{E}_{P}\left[\nu_{\omega}(i t)\left(f_{\omega}\right) f_{\rho}^{\omega}(t)\right]=\mathbb{E}_{P}\left[\mu_{\omega}\left(h_{\omega}(i t)\right) f_{1, \rho}^{\omega}(t)\right]
$$

where

$$
f_{1, \rho}^{\omega}(t)=\sum_{n \geq 1} \rho^{n-1} \lambda_{\theta^{-n} \omega}(i t) \nu_{\theta^{-n} \omega}(i t)\left(f_{\theta^{-n} \omega}\right) .
$$

Therefore, for any function $g_{1}$ with the properties described at the beginning of Section 4.7

$$
V_{\rho}\left(g_{1}\right)=\int_{-\delta_{0}}^{\delta_{0}} e_{1}(t) \mathbb{E}_{P}\left[\mu_{\omega}\left(h_{\omega}(i t)\right) f_{1, \rho}^{\omega}(t)\right] d t
$$

where in the non-lattice case $e_{1}(t)=\hat{g}_{1}(t)$, while in the lattice case $e_{1}(t)=\sum_{k} \hat{g}_{1}(t+$ $\left.\frac{2 \pi k}{h}\right)$. Set $\tilde{\Gamma}_{n}=\theta^{-n} \Gamma_{n}$. Repeating the arguments of the proof of Lemma 4.5 with $\lambda_{\omega}(i t), f_{1, \rho}^{\omega}(t)-f_{1, \rho}^{\theta \omega}(t), \lambda_{\theta^{-n} \omega, n}(i t), \tilde{\Gamma}_{n}$ and $\nu_{\theta^{-n} \omega}(i t)\left(f_{\theta^{-n} \omega}\right)$ in place of $\lambda_{\theta^{-1} \omega}(i t)$, $f_{\rho}^{\omega}(t)-f_{\rho}^{\theta^{-1} \omega}(t), \lambda_{\omega, n}(i t), \Gamma_{n}$ and $\mu_{\theta^{n} \omega}\left(h_{\theta^{n} \omega}(i t)\right)$, respectively, we obtain (2.9) in the case when $\nu_{\omega}^{\prime}(0)\left(f_{\omega}\right)$ does not depend on $\omega$.

6.4. Markov chains with transition densities. Let $(\Omega, \mathcal{F}, P, \theta)$ and $(\mathcal{X}, \rho), \mathcal{E} \subset$ $\Omega \times \mathcal{X}$ and $\mathcal{E}_{\omega}$ satisfy the conditions specified in Section 6.2. For any $\omega \in \Omega$ denote by $B_{\omega}$ the Banach space of all bounded Borel functions $g: \mathcal{E}_{\omega} \rightarrow \mathbb{C}$ together with the supremum norm $\|\cdot\|_{\infty}$. For any $g: \mathcal{E} \rightarrow \mathbb{C}$ consider the functions $g_{\omega}: \mathcal{E}_{\omega} \rightarrow \mathbb{C}$ given by $g_{\omega}(x)=g(\omega, x)$. Then by Lemma 5.1 .3 in [13, the norm $\omega \rightarrow\left\|g_{\omega}\right\|_{\infty}$ is a $\mathcal{F}$-measurable function of $\omega$, for any measurable $g: \mathcal{E} \rightarrow \mathbb{C}$.

Let $r_{\omega}=r_{\omega}(x, y): \mathcal{E}_{\omega} \times \mathcal{E}_{\theta \omega} \rightarrow[0, \infty), \omega \in \Omega$ be a family of integrable in $y$ Borel measurable functions, $m_{\omega}, \omega \in \Omega$ be a family of Borel probability measures 
on $\mathcal{E}_{\omega}$ and $u: \mathcal{E} \rightarrow \mathbb{R}$ be a measurable function so that $u_{\omega} \in B_{\omega}, P$-a.s. and that the random variable $\sup \left|u_{\omega}\right|=\left\|u_{\omega}\right\|_{\infty}$ is bounded. Consider the family of random operators $R_{z}^{\omega}, z \in \mathbb{C}$ which map (bounded) Borel functions $g$ on $\mathcal{E}_{\theta \omega}$ to Borel measurable functions on $\mathcal{E}_{\omega}$ by the formula

$$
R_{z}^{\omega} g(x)=\int_{\mathcal{E}_{\theta \omega}} r_{\omega}(x, y) e^{z u_{\theta \omega}(y)} g(y) d m_{\theta \omega}(y) .
$$

We will assume that $R_{0}^{\omega}$ are Markov operators, namely that $R_{0}^{\omega} \mathbf{1}=\mathbf{1}$ where $\mathbf{1}$ is the function which takes the constant value 1 on $\mathcal{E}_{\theta \omega}$. Observe that

$$
\left\|R_{0}^{\omega}\right\|_{\infty}:=\sup _{g \in B_{\theta \omega}:\|g\|_{\infty} \leq 1}\left\|R_{0}^{\omega} g\right\|_{\infty}=\left\|R_{0}^{\omega} \mathbf{1}\right\|_{\infty}
$$

and therefore for $P$-a.a. $\omega$ we have $\left\|R_{z}^{\omega}\right\|_{\infty}<\infty$ for any $z \in \mathbb{C}$, namely, $R_{z}^{\omega}$ is a continuous linear operator between the Banach spaces $B_{\theta \omega}$ and $B_{\omega}$.

6.3. Assumption. The maps $\omega \rightarrow \int_{\mathcal{E}_{\omega}} g_{\omega}(x) d m_{\theta \omega}(x)$ and $(\omega, x) \rightarrow R_{0}^{\omega} g_{\theta \omega}(x)$, $(\omega, x) \in \mathcal{E}$ are measurable for any bounded measurable function $g: \mathcal{E} \rightarrow \mathbb{C}$.

For any $\omega \in \Omega, n \in \mathbb{N}$ and $z \in \mathbb{C}$ consider the $n$-th order iterates $R_{z}^{\omega, n}: B_{\theta^{n} \omega} \rightarrow$ $B_{\omega}$ given by

$$
R_{z}^{\omega, n}=R_{z}^{\omega} \circ R_{z}^{\theta \omega} \circ \cdots \circ R_{z}^{\theta^{n-1} \omega}
$$

Then we can write

$$
R_{0}^{\omega, n} g(x)=\int_{\mathcal{E}_{\theta^{n} \omega}} r_{\omega}(n, x, y) g(y) d m_{\theta^{n} \omega}(y)
$$

for some family $r_{\omega}(n, \cdot, \cdot)=r_{\omega}(n, x, y): \mathcal{E}_{\omega} \times \mathcal{E}_{\theta^{n} \omega} \rightarrow[0, \infty)$ of integrable in $y$ Borel measurable functions. We will assume that the following random version of the two sided Doeblin condition holds true.

6.4. Assumption. There exist a bounded random variable $j_{\omega} \in \mathbb{N}$ and $\alpha_{m}(\omega) \geq 1$, $m \in \mathbb{N}$ such that $P$-a.s.,

$$
\alpha_{m}(\omega) \leq r_{\omega}(m, x, y) \leq\left(\alpha_{m}(\omega)\right)^{-1}
$$

for any $m \geq j_{\omega}, x \in \mathcal{E}_{\omega}$ and $y \in \mathcal{E}_{\theta^{m} \omega}$. Moreover, let $j_{0}$ be so that $j_{\omega} \leq j_{0}, P$-a.s. Then there exists $\alpha>0$ so that $\alpha_{n}(\omega) \geq \alpha$ for any $j_{0} \leq n \leq 2 j_{0}$.

Let $\mu_{\omega}$ be a (measurable in $\omega$ ) probability measure on $\mathcal{E}_{\omega}$ and let $\xi_{n}^{\theta^{n}} \omega, n \geq 1$ be a Markov chain with initial distribution $\mu_{\omega}$ whose $n$-th step operator is given by $R_{0}^{\omega, n}$. Set

$$
S_{n}^{\omega}=\sum_{j=0}^{n-1} u_{\theta^{j} \omega}\left(\xi_{j}^{\theta^{j} \omega}\right) .
$$

Let $S_{n}$ be the random variable generated by drawing $\omega$ according to $P$ and taking on the fibers the distribution of $S_{n}^{\omega}$, namely the random variable whose characteristic function is given by

$$
\mathbb{E} e^{i t S_{n}}=\int \mu_{\omega}\left(R_{i t}^{\omega, n} \mathbf{1}\right) d P(\omega) .
$$

Under Assumption 6.4, in [22] the author proved that the limit $\sigma^{2}=$ $\lim _{n \rightarrow \infty} n^{-1} \operatorname{var} S_{n}^{\omega}$ exists $P$-a.s., and it does not depend on $\omega$.

Next, let $\omega_{0} \in \Omega$ and $n_{0} \in \mathbb{N}$ be so that $\theta^{n_{0}} \omega_{0}=\omega_{0}$. We will call the case the non-lattice case if for any $t \in \mathbb{R} \backslash\{0\}$ the spectral radius of the operator $R_{i t}^{\omega_{0}, n_{0}}$ is 
strictly less than 1 . We will call the case a lattice one if for some $h>0$ the function $u$ takes values on the lattice $h \mathbb{Z}$ and the spectral radius of the operators $R_{i t}^{\omega_{0}, n_{0}}, t \in$ $\left(-\frac{2 \pi}{h}, \frac{2 \pi}{h}\right) \backslash\{0\}$ are strictly less than 1. We refer to [15] for a characterization of these lattice and non-lattice cases which resembles the description of these cases in the transfer operator case.

6.5. Theorem. Suppose that Assumptions 6.3 and 6.4 hold true, that $\sigma^{2}>0$ and that $\gamma=\mu_{\omega}\left(u_{\omega}\right)$ does not depend on $\omega$. Then $\sigma^{-1} n^{-\frac{1}{2}}\left(S_{n}-n \gamma\right), n \geq 1$ converges in distribution as $n \rightarrow \infty$ towards the standard normal law, and $S_{n}-n \gamma, n \geq 1$ satisfies the appropriate LLT (in both lattice and non-lattice cases). Moreover, when $\gamma>0$ then all the statements in Theorem 6.2 hold true.

6.6. Remark. In the above integral operator case it is possible to obtain similar limit theorems without using Assumption 2.5 and (2.8), relying instead on some assumption on the distribution of the process $j_{\theta^{k} \omega}, k \geq 1$ in the spirit of (2.7). In 12 we proved a local limit theorem for certain "nonconventional" sums. Our proof there was based on a certain reduction to a problem of bounding expectations of norms of iterates of random Fourier operators (the proof was in the spirit of the argument in [26]). This is exactly the situation of annealed limit theorems, and so, similar to 12 argument will yield the desired results.

\section{REFERENCES}

[1] R. Aimino, M. Nicol and S. Vaienti. Annealed and quenched limit theorems for random expanding dynamical systems, Probab. Th. Rel. Fields 162, 233-274, (2015).

[2] J.R. Blum, D.L. Hanson and L.H. Koopmans, On the strong law of large numbers for a class of stochastic processes, Z. Wahrsch. verw. Geb 2 (1963), 1-11.

[3] R. Bowen, Equilibrium states and the ergodic theory of Anosov diffeomorphisms, Lecture Notes in Mathematics, volume 470, Springer Verlag, 1975.

[4] L. Breiman, Probability, SIAM, Philadelphia (1992).

[5] J.R. Chazottes and S. Gouëzel, Optimal concentration inequalities for dynamical systems, Comm. Math. Phys. 316 (2012), 843-889.

[6] J.R. Chazottes, Fluctuations of observables in dynamical systems: from limit theorems to concentration inequalities, Nonlinear dynamics new directions, Vol. 11, Nonlinear Syst. Complex., pages 4785. Springer, 2015.

[7] C. Castaing and M.Valadier, Convex analysis and measurable multifunctions, Lecture Notes Math., vol. 580, Springer, New York, 1977.

[8] J. Dedecker, P. Doukhan, G. Lang, J.R. Len, S. Louhichi, C. Prieur, Weak Dependence: With Examples and Applications, Lecture Notes in Statistics, vol 190, Springer-Verlag, Berlin (2007).

[9] P. Doukhan and M.Neumann, Probability and moment inequalities for sums of weakly dependent random variables, with applications, Stochastic Process. Appl. 117 (2007), 878-903.

[10] D. Dragičević, G. Froyland, C. González-Tokman and S. Vaienti, A spectral approach for quenched limit theorems for random expanding dynamical systems, Commun. Math. Phys. 360, 1121-1187 (2018).

[11] Y. Guivarćh and J. Hardy, Théorèmes limites pour une classe de chaînes de Markov et applications aux difféomorphismes d'Anosov, Ann. Inst. H. Poincaré Probab. Statist. 24 (1988), no. 1, 73-98.

[12] Y. Hafouta and Yu. Kifer, A nonconventional local limit theorem, J. Theor. Probab. 29 (2016), 1524-1553.

[13] Y. Hafouta and Yu. Kifer, Nonconventional limit theorems and random dynamics, World Scientific, Singapore, 2018. 
[14] Y. Hafouta, Nonconventional moderate deviations theorems and exponential concentration inequalities, arXiv preprint 1805.00849.

[15] H. Hennion and L. Hervé, Limit Theorems for Markov Chains and Stochastic Properties of Dynamical Systems by Quasi-Compactness, Lecture Notes in Mathematics vol. 1766, Springer, Berlin, 2001.

[16] N.T.A. Haydn and Y. Psiloyenis, Return times distribution for Markov towers with decay of correlations, Nonlinearity 27 (2014), 1323-1349.

[17] N.T.A. Haydn and F. Yang, Local escape rates for $\phi$-mixing dynamical systems, arXiv preprint 1806.07148.

[18] H. Ishitani, Central limit theorems for the random iterations of 1-dimensional transformations (Dynamics of complex systems), RIMS Kokyuroku, 1404, 21-31, (2004).

[19] R.S. Kallabis and M.H. Neumann, An exponential inequality under weak dependence, Bernoulli 12 (2006) 333-350.

[20] Yu. Kifer, Large deviations for random expanding maps, Lyapunov Exponents, eds. L. Arnold, H. Crauel and J.P. Eckmann (Springer-Verlag, 1991), pp. 178-186.

[21] Yu. Kifer, Perron-Frobenius theorem, large deviations, and random perturbations in random environments, Math. Z. 222(4) (1996), 677-698.

[22] Yu. Kifer, Limit theorems for random transformations and processes in random environments, Trans. Amer. Math. Soc. 350 (1998), 1481-1518.

[23] Yu. Kifer, Thermodynamic formalism for random transformations revisited, Stoch. Dyn. 8 (2008), 77-102.

[24] V. Mayer, B. Skorulski and M. Urbański, Distance expanding random mappings, thermodynamical formalism, Gibbs measures and fractal geometry, Lecture Notes in Mathematics, vol. 2036 (2011), Springer.

[25] S.V. Nagaev, Some limit theorems for stationary Markov chains, Theory Probab. Appl. 2 (1957), 378-406.

[26] S.V. Nagaev, More exact statements of limit theorems for homogeneous Markov chains, Theory Probab. Appl. 6 (1961), 62-81.

[27] L. Saulis and V.A. Statulevicius, Limit Theorems for Large Deviations, Kluwer Academic, Dordrecht, Boston, 1991.

[28] L.S. Young, Statistical properties of dynamical systems with some hyperbolicity, Ann. Math. 7 (1998) 585-650.

[29] L.S. Young, Recurrence time and rate of mixing, Israel J. Math. 110 (1999) 153-88.

Institute of Mathematics, The Hebrew University, Jerusalem 91904, Israel

E-mail address: yeor.hafouta@mail.huji.ac.il 